\title{
Checklist of British and Irish Hymenoptera - Sawflies, 'Symphyta'
}

\author{
Andrew D. Liston ${ }^{\dagger}$, Guy T. Knight ${ }^{\ddagger}$, David A. Sheppard§, Gavin R. Broad', Laurence Livermorel \\ † Senckenberg Deutsches Entomologisches Institut, Müncheberg, Germany \\ $\ddagger$ Entomology, National Museums Liverpool, Liverpool, United Kingdom \\ $\S 32$ South Street, Alford, Lincolnshire, United Kingdom \\ I The Natural History Museum, London, United Kingdom
}

\author{
Corresponding author: Andrew D. Liston (andrew.liston@senckenberg.de), Guy T. Knight \\ (guy.knight@liverpoolmuseums.org.uk), Gavin R. Broad (g.broad@nhm.ac.uk), Laurence Livermore \\ (laurence.livermore@gmail.com) \\ Academic editor: Jose Fernandez-Triana \\ Received: 14 Jul 2014 | Accepted: 11 Aug 2014 | Published: 29 Aug 2014 \\ Citation: Liston A, Knight G, Sheppard D, Broad G, Livermore L (2014) Checklist of British and Irish \\ Hymenoptera - Sawflies, 'Symphyta'. Biodiversity Data Journal 2: e1168. doi: 10.3897/BDJ.2.e1168 \\ ZooBank: urn:Isid:zoobank.org:pub:3C3EC7B0-9BA1-4584-8E3E-89CBBD28B432
}

\section{Introduction}

The superfamilies Cephoidea, Orussoidea, Pamphilioidea, Siricoidea, Tenthredinoidea, Xiphydrioidea and Xyeloidea are combined as one checklist section, as the sawflies represent a distinctive assemblage of phytophagous (except Orussidae) Hymenoptera. More than fifty years have passed since the publication of the final part of Robert B. Benson's three part identification key to the "Symphyta" of the British Isles (Benson 1935, Benson 1952, Benson 1958). Whilst the first two parts were based substantially on the acute monography by Eduard Enslin (Enslin 1912, Enslin 1913, Enslin 1914, Enslin 1915, Enslin 1916, Enslin 1917 and Enslin 1918), Benson's treatment of the Nematinae represented an original and significant step forward in our knowledge of this subfamily. The results of subsequent research on the British and Irish sawfly fauna were collated by Quinlan (1978). Since then, renewed investigation of the taxonomy of West Palaearctic sawflies has led to a great number of taxonomic and nomenclatural changes, affecting many taxa occurring in the British Isles.

Of particular value in accessing the extensive but widely scattered literature on sawflies, is the online database "Electronic World Catalog of Symphyta (ECatSym)" (Blank et al. 
2012). Unfortunately, the only identification guide to the European "Symphyta" published since 1958 with a more than local geographical scope, by Zhelochovtsev (1988), suffers from weaknesses, such as the reliance placed on the opinions and illustrations of $E$. Lindqvist in its treatment of the Nematinae. Mistakes in translation from Russian make the later English language edition of this work even more difficult to use.

Special problems are attached to the interpretation of records for some species not found in the British Isles since their inclusion in the works of Leach (1817) and Stephens (1835). Some of these are probably extinct, but their presence in the British Isles has in many cases never been unequivocally proved: see for example Benson (1943). We therefore maintain the practice, as in Fitton et al. (1978), of marking names of such taxa with a preceding "?". Further, the taxonomic status of some of the nominal taxa described from the British Isles by Hill, Stephens, Newman and Cameron is still unclear, particularly where no type material has been located. The number of confirmed British sawfly species now stands at 537, compared to 471 in the 1978 checklist (Quinlan 1978; Broad 2014). Note that, since the publication of the introduction section (Broad 2014) a further family, Heptamelidae, has been recognised (Malm and Nyman 2014), including two British species previously included in Tenthredinidae.

Authorship and date of publication of original descriptions by various authors follow mainly the bibliographic research of Taeger and Blank (1996), Taeger and Blank (2006), Blank and Taeger (1998), Blank et al. (2009) and Taeger et al. (2010).

The classification of genera used here is largely based on the system of Benson (1951-1958). This has the advantages of being relatively simple and widely known. Detailed phylogenetic studies on several major lineages of Tenthredinidae are still lacking. However, some definite conclusions on generic groupings were reached by Nyman et al. (2006) on the Nematinae and Leppänen et al. (2012) on the Fenusini of the Blennocampinae. For the higher Nematinae, the full taxonomic and nomenclatural consequences of the recently proposed phylogeny have not yet been drawn. Far fewer genera will in future be recognised. For the present, we revert here to a generic classification that is similar to that used by Benson (1958), even though we are aware that Pachynematus, for example, is not monophyletic.

Genus and species group names are included selectively in the synonymy. For a complete current synonymy of all these, including important misidentifications, see ECatSym (Blank et al. 2012). A name is only included as a synonym when it fulfils one or both of the following criteria:

- $\quad$ has at some time been used for a taxon occurring in the British Isles;

- is used in widely consulted works on taxonomy or distribution, or in original accounts of biological characters / descriptions of immature stages.

The general scope and rationale for the checklist are covered by Broad (2014). As for the rest of the checklist, the Channel Islands fauna is excluded: see Sheppard (1990) on the sawfly fauna of the Channel Islands. There is a lack of data on the sawflies of the Isle of 
Man. It should be noted that several species were mistakenly listed as occurring in Scotland by Liston (1995).

Some of the diversity of British sawflies is illustrated in Figs 1, 2, 3, 4.
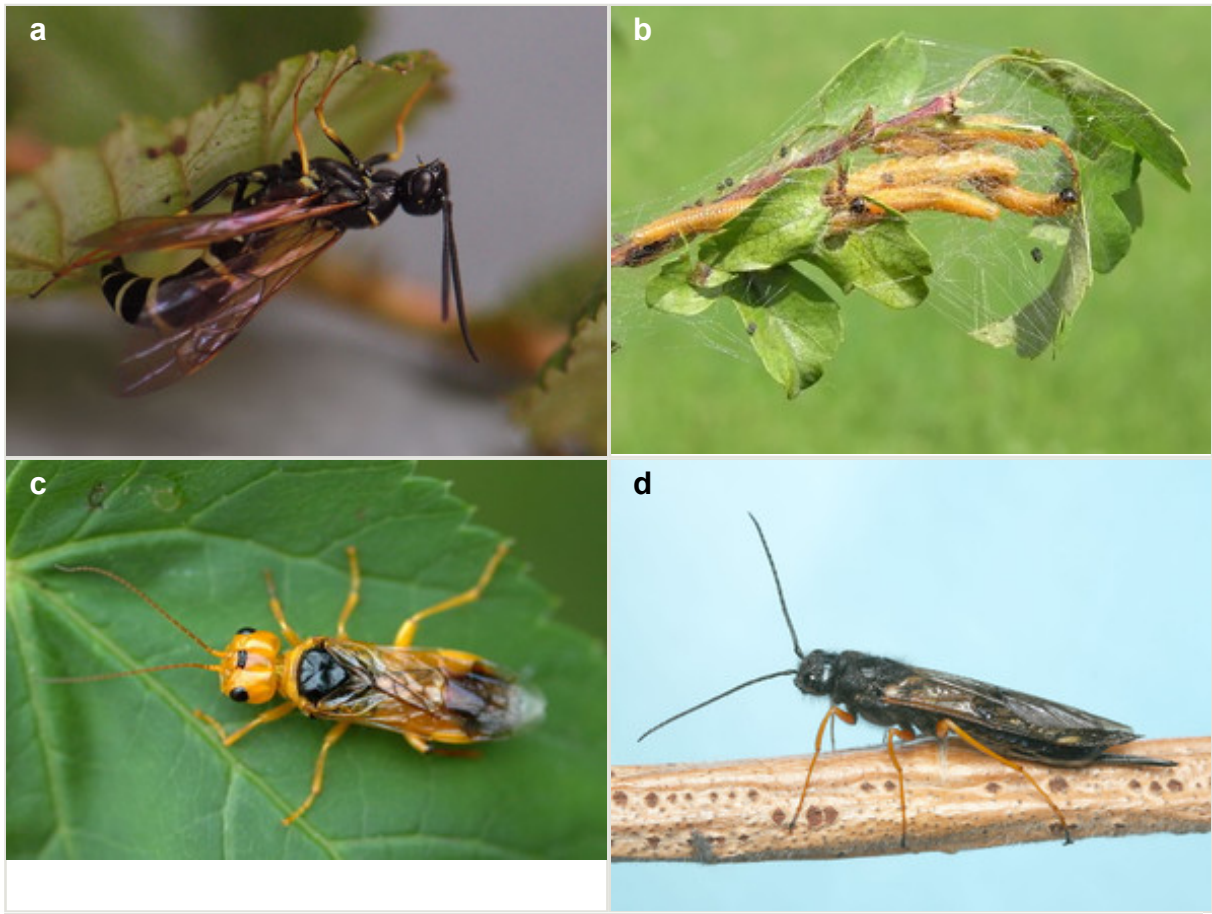

Figure 1.

Representative British sawflies, superfamilies Cephoidea, Pamphilioidea and Siricoidea.

a: Cephidae, Phylloecus xanthostoma (G. Knight)

b: Pamphiliidae, Neurotoma saltuum larvae (G. Nowotny)

c: Pamphiliidae, Pamphilius betulae (H. Lewerenz)

d: Siricidae, Sirex noctilio (H. Goulet) 

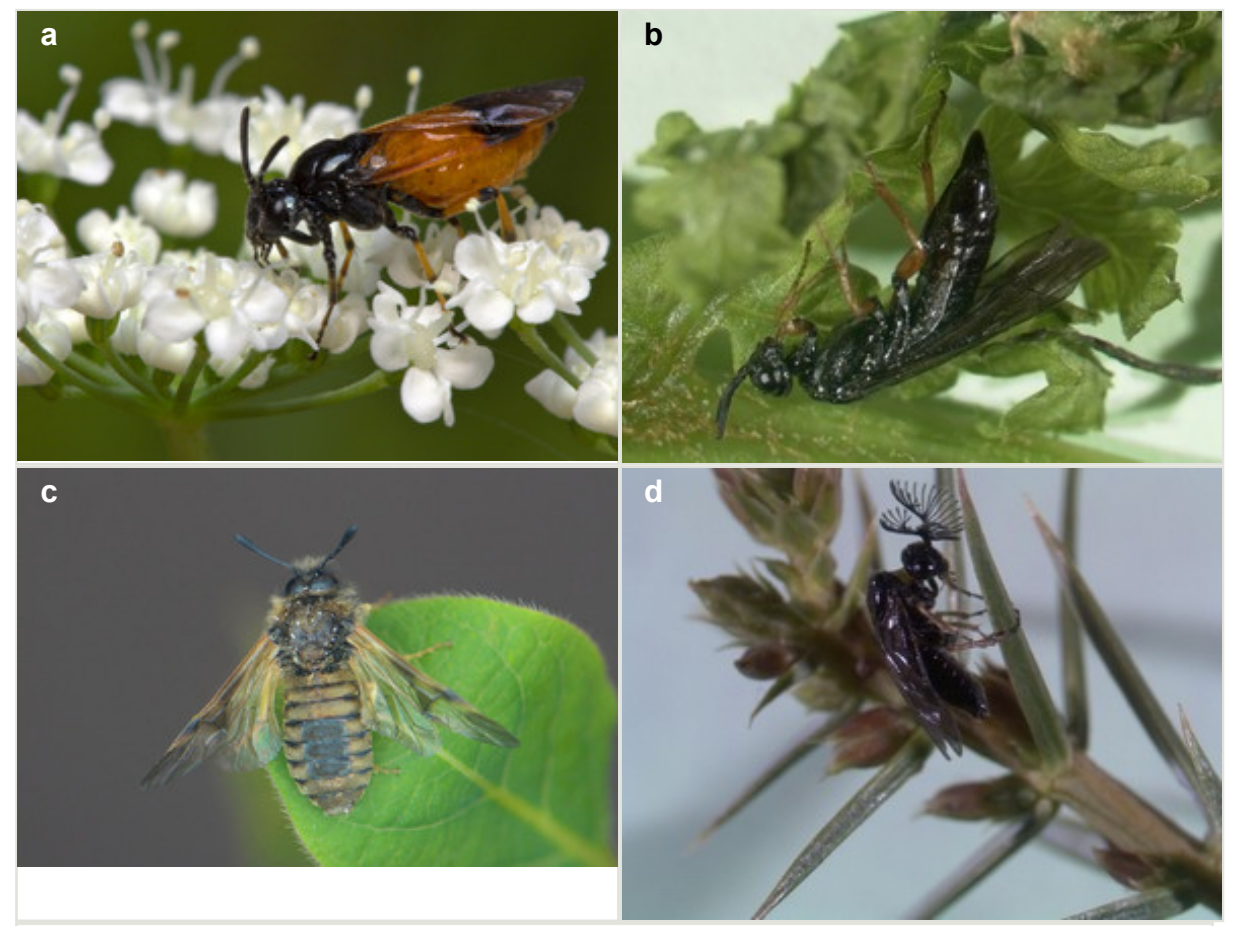

Figure 2.

Representative British sawflies, superfamily Tenthredinoidea.

a: Argidae, Arge cyanocrocea (T. Tarvainis)

b: Blasticotomidae, Blasticotoma filiceti (A. Liston)

c: Cimbicidae, Abia lonicerae (G. Knight)

d: Diprionidae, Monoctenus juniperi (A. Taeger) 

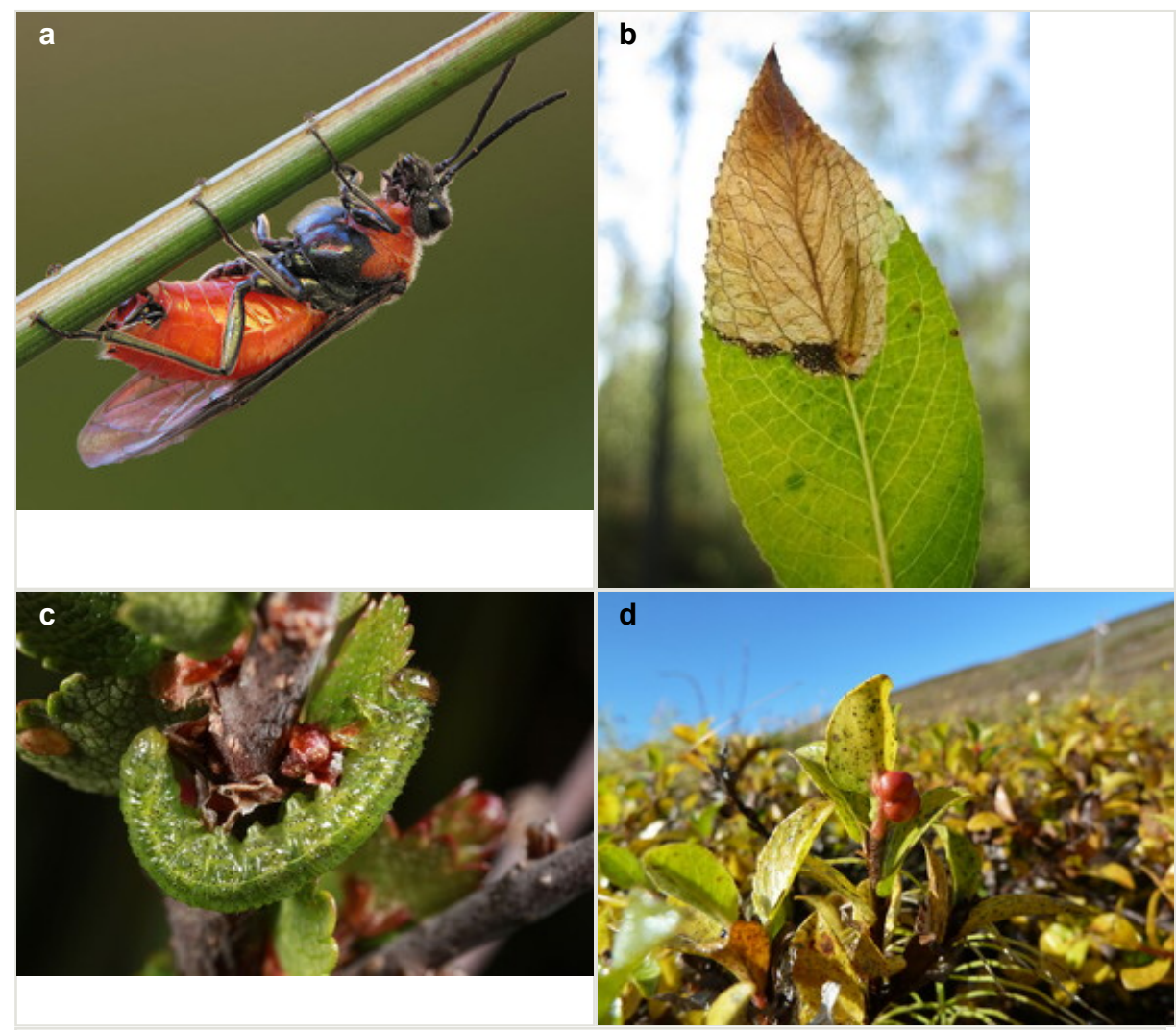

Figure 3.

Representative British sawflies, superfamily Tenthredinoidea.

a: Tenthredinidae, Dolerus madidus (T. Thieme)

b: Tenthredinidae, Heterarthrus microcephalus larval mine (T. Nyman)

c: Tenthredinidae, Amauronematus tristis larva (A. Watson-Featherstone)

d: Tenthredinidae, Pontania myrsiniticola gall (A. Liston) 

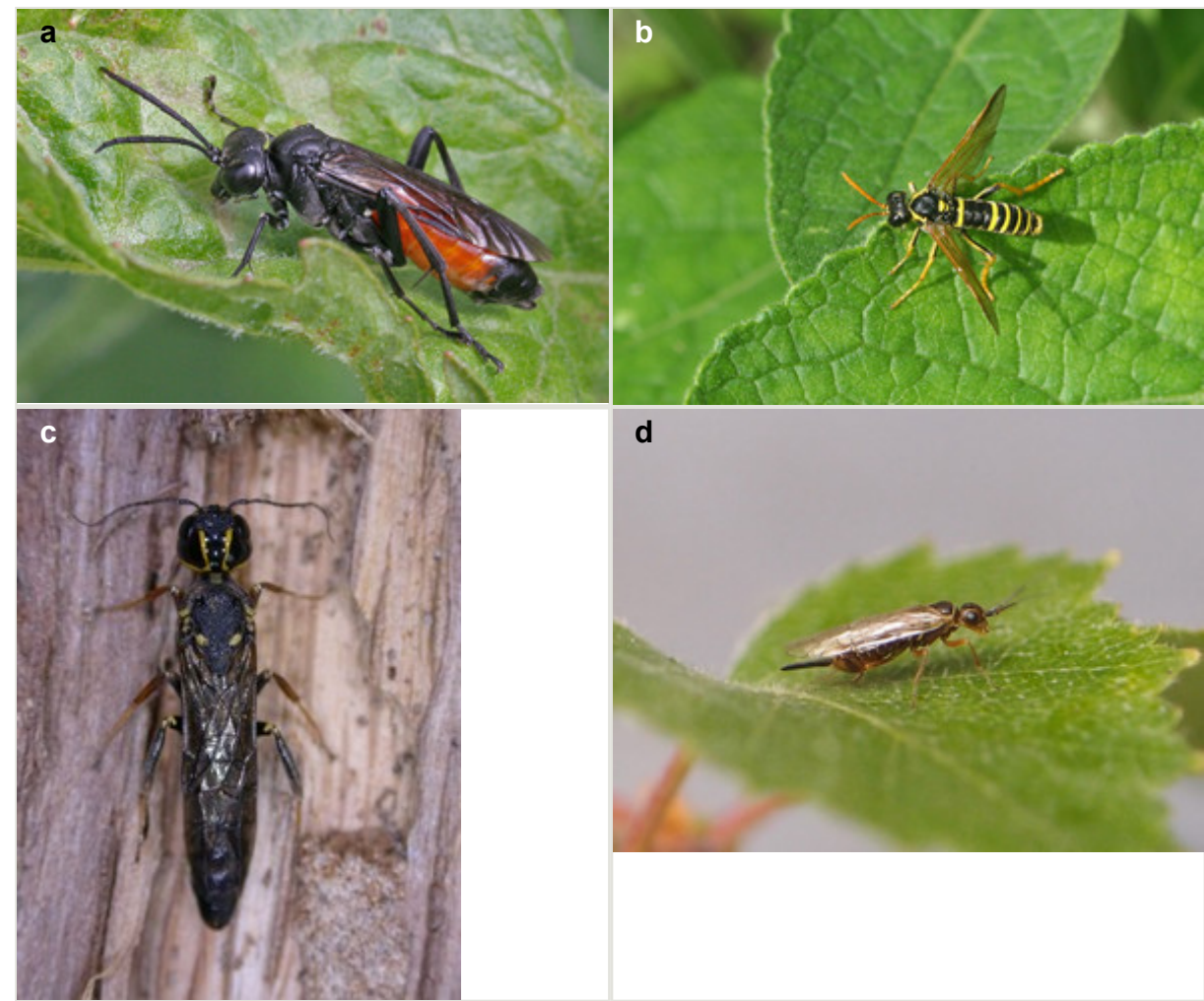

d

Figure 4.

Representative British sawflies, superfamilies Tenthredinoidea, Xiphydrioidea and Xyeloidea.

a: Tenthredinidae, Macrophya annulata (U. Rindlisbacher)

b: Tenthredinidae, Tenthredo scrophulariae (H. Savina)

c: Xiphydriidae, Xiphydria longicollis (F. Stergulc)

d: Xyelidae, Xyela curva (G. Knight) 


\section{Superfamily CEPHOIDEA Newman, 1834}

Family Cephidae Newman, 1834

Subfamily Cephinae Newman, 1834

Tribe Cephini Newman, 1834

Genus Calameuta Konow, 1896

Nomenclature:

MONOPLOPUS Konow, 1896

HAPLOCEPHUS Benson, 1935

\section{Calameuta filiformis (Eversmann, 1847)}

Nomenclature:

Cephus filiformis Eversmann, 1847

elongatus (Vollenhoven, 1858, Cephus)

arundinis (Giraud, 1863, Cephus)

Cephus analis (Klug, 1803): Stephens, 1835 misident.

Distribution: England, Wales

Calameuta pallipes (Klug, 1803)

Nomenclature:

Astatus pallipes Klug, 1803

pusilla (Stephens, 1835, Cephus)

immaculata (Stephens, 1835, Cephus)

cultrarius (Hartig, 1837, Cephus)

pallidipes (Dalla Torre, 1894, Cephus)

phthisica (Fabricius, 1805, Cephus): misspelling

Distribution: England, Scotland, Wales, Ireland 
Genus Cephus Latreille, 1802

Cephus nigrinus Thomson, 1871

Nomenclature:

pallipes (Klug, 1803): Stephens, 1835 misident.

Distribution: England

Cephus pygmeus (Linnaeus, 1767)

Nomenclature:

Sirex pygmeus Linnaeus, 1767

floralis (Klug, 1803, Astatus)

atripes Stephens, 1835

pygmaeus: misspelling

Distribution: England, Wales

Cephus spinipes (Panzer, 1800)

Nomenclature:

Banchus spinipes Panzer, 1800

pilosulus Thomson, 1871

pusillus: Stephens, 1835 misident.

cultratus: Eversmann, 1847 misident. and misspelling of cultrarius Hartig

Distribution: England, Wales

Genus Trachelus Jurine, 1807

Nomenclature:

CEPHA Billberg, 1820

ATEUCHOPUS Konow, 1896

EUMETABOLUS Schulz, 1906

TRACHELASTATUS Morice \& Durrant, 1915

NEATEUCHOPUS Benson, 1935

MICROCEPHUS Benson, 1935 
Trachelus tabidus (Fabricius, 1775)

Nomenclature:

Sirex tabidus Fabricius, 1775

macilentus (Fabricius, 1793, Sirex)

mandibularis (Serville, 1823, Cephus)

nigritus (Serville, 1823, Cephus)

Distribution: England, Wales

Trachelus troglodyta (Fabricius, 1787)

Nomenclature:

Sirex troglodyta Fabricius, 1787

niger (Harris, 1779): Cameron, 1890 misident.

Distribution: †England

Notes: Extinct in Britain

Tribe Hartigiini Enslin, 1914

Genus Janus Stephens, 1829

Nomenclature:

EPHIPPIONOTUS Costa, 1860

Janus cynosbati (Linnaeus, 1758)

Nomenclature:

Tenthredo cynosbati Linnaeus, 1758

femoratus (Curtis, 1830, Cephus)

niger (Brischke, 1892, Cephus)

Distribution: England

Notes: Nomenclature follows Blank et al. (2009).

Janus luteipes (Lepeletier, 1823)

Nomenclature:

Cephus luteipes Lepeletier, 1823

connectens Stephens, 1829 
bifrons Newman, 1838

Distribution: England, Wales

Genus Phylloecus Newman, 1838

Nomenclature:

HARTIGIA Schiødte, 1839

MACROCEPHUS Schlechtendal, 1878

COPIOSOMA W. F. Kirby, 1882

ADIRUS Konow, 1899

Notes: Synonymy of Hartigia under Phylloecus is discussed by Liston and Prous (2014).

Phylloecus faunus Newman, 1838

Nomenclature:

helleri (Taschenberg, 1871, Cephus)

albomaculatus (J. Stein, 1876, Cephus)

Distribution: England

Notes: Added by Liston and Prous (2014) on the basis of the lectotype in the collections of Oxford University Museum of Natural History that Newman (1838) stated was collected "in the vicinity of London".

\section{Phylloecus linearis (Schrank, 1781)}

Nomenclature:

Tenthredo linearis Schrank, 1781

quinquefasciata (Stephens, 1835, Cephus)

major (Eversmann, 1847, Cephus)

agrimoniae (Goury, 1912, Macrocephus)

Distribution: England, Wales

Phylloecus niger (M. Harris, 1779)

Nomenclature:

Sirex niger M. Harris, 1779

satyrus (Panzer, 1801, Astatus)

brachyptera (Damianitsch, 1866, Cephus) 
Distribution: England, Wales

Phylloecus xanthostoma (Eversmann, 1847)

Nomenclature:

Cephus xanthostoma Eversmann, 1847

ulmariae (Schlechtendal, 1878, Macrocephus)

Distribution: England, Scotland, Wales

\section{Superfamily ORUSSOIDEA Newman, 1834}

Family Orussidae Newman, 1834

Genus Orussus Latreille, 1797

Nomenclature:

ORYSSUS Fabricius, 1798

Orussus abietinus (Scopoli, 1763)

Nomenclature:

Sphex abietina Scopoli, 1763

Distribution: ?England

Notes: Recorded from Kent and Devon by Stephens (1835), but see comments in Benson (1951).

\section{Superfamily PAMPHILIOIDEA Cameron, 1890}

Family Megalodontesidae Konow, 1897

Nomenclature:

Megalodontidae preocc.

Genus Megalodontes Latreille, 1802

Nomenclature:

TARPA Fabricius, 1804 
Notes: If any member of this genus was ever present in England, then only $M$. cephalotes seems at all likely: according to Leach (1817) and Stephens (1835), found near Bristol and Plymouth. See Taeger (2002) for current taxonomy. of the genus.

Species of Megalodontes removed from the British and Irish list:

[panzeri (Leach, 1817), plagiocephalus (Fabricius, 1804)] Up to three separate Megalodontes species have at times been considered to have been present in England. Neither M. panzeri nor $M$. plagiocephalus, on the basis of their currently known distributions and habitat requirements, probably ever occurred in the British Isles.

Megalodontes cephalotes (Fabricius, 1781)

Nomenclature:

Tenthredo cephalotes Fabricius, 1781

klugii (Leach, 1817, Tarpa)

spissicornis (Klug, 1824, Tarpa)

klugi: misspelling

Distribution: ?England

Family Pamphiliidae Cameron, 1890

Notes: Unplaced species of Pamphiliidae, treated as nomen oblitum by Blank et al. (2009):

Tenthredo variegata Hill, 1773

Family Cephalciinae Benson, 1945

Genus Acantholyda Costa, 1894

Nomenclature:

ITYCORSIA Konow, 1897

Acantholyda erythrocephala (Linnaeus, 1758)

Nomenclature:

Tenthredo erythrocephala Linnaeus, 1758

Distribution: England, Scotland 


\section{Acantholyda posticalis (Matsumura, 1912)}

Nomenclature:

Lyda posticalis Matsumura, 1912

stellata (Christ, 1791, Tenthredo) preocc.

?pinivora Enslin, 1918

nemoralis (Linnaeus, 1758): Thomson, 1871 misident.

Distribution: England, Scotland

\section{Genus Cephalcia Panzer, 1803}

\section{Nomenclature:}

CEPHALCIA Jurine, 1801 suppressed

CEPHALEIA Jurine, 1807

Notes: Species of Cephalcia removed from the British and Irish list:

[arvensis Panzer, 1803] Recorded by Shinohara (2002) but deleted by Shaw (2003) because material probably not of British origin.

\section{Cephalcia lariciphila (Wachtl, 1898)}

Nomenclature:

Cephaleia lariciphila Wachtl, 1898

alpina (Klug, 1808): misident.

falleni (Dalman, 1823): misident.

annulata (Hartig, 1837): misident.

Distribution: England, Scotland, Wales

Subfamily Pamphiliinae Cameron, 1890

Genus Neurotoma Konow, 1897

Nomenclature:

GONGYLOCORSIA Konow, 1897

GONGYLOCORISA: Ashmead, 1898 misspelling 
Neurotoma mandibularis (Zaddach, 1866)

Nomenclature:

Lyda mandibularis Zaddach, 1866

Distribution: England

Neurotoma saltuum (Linnaeus, 1758)

Nomenclature:

Tenthredo saltuum Linnaeus, 1758

flaviventris (Retzius, 1783, Tenthredo)

fasciata (Curtis, 1831, Lyda)

Distribution: England, Wales

Genus Pamphilius Latreille, 1802

Nomenclature:

LYDA Fabricius, 1804

ANOPLOLYDA Costa, 1894

BACTROCERUS Konow, 1897

Notes: Species of Pamphilius removed from the British and Irish list:

[jucundus (Eversmann, 1847, Lyda) (nemorum auctt.)] See Liston et al. (2006)

Pamphilius albopictus (Thomson, 1871)

Nomenclature:

Lyda albopicta Thomson, 1871

Distribution: Scotland

Notes: Added by Shinohara (1998)

Pamphilius balteatus (Fallén, 1808)

Nomenclature:

Lyda balteata Fallén, 1808

cingulatus Latreille, 1812

Distribution: England, Scotland, Wales, Ireland 
Pamphilius betulae (Linnaeus, 1758)

Nomenclature:

Tenthredo betulae Linnaeus, 1758

Distribution: England

Pamphilius fumipennis (Curtis, 1831)

Nomenclature:

Lyda fumipennis Curtis, 1831

Distribution: England, Wales

Pamphilius gyllenhali (Dahlbom, 1835)

Nomenclature:

See ICZN (2005) Opinion 2101.

Lyda gyllenhali Dahlbom, 1835

Distribution: England, Scotland, Wales, Ireland

Pamphilius histrio Latreille, 1812

Nomenclature:

flaviventris (Retzius, 1783): Stephens, 1835 misident.

Distribution: England, Scotland, Wales

Pamphilius hortorum (Klug, 1808)

Nomenclature:

Lyda hortorum Klug, 1808

hortorum ssp. bicinctus Benson, 1945

Distribution: England, Scotland, Wales, Ireland

Pamphilius inanitus (Villers, 1789)

Nomenclature:

Tenthredo inanita Villers, 1789

Distribution: England, Scotland, Wales, Ireland 
Pamphilius latifrons (Fallén, 1808)

Nomenclature:

See ICZN (2005), Opinion 2101.

Lyda latifrons Fallén, 1808

maculosus (Zaddach, 1866, Lyda)

Distribution: England

Pamphilius pallipes (Zetterstedt, 1838)

Nomenclature:

Lyda pallipes Zetterstedt, 1838

Distribution: England, Scotland, Wales, Ireland

Pamphilius stramineipes (Hartig, 1837)

Nomenclature:

Lyda stramineipes Hartig, 1837

arbustorum (Fabricius, 1793): Cameron, 1885 misident.

Distribution: Scotland

Pamphilius sylvarum (Stephens, 1835)

Nomenclature:

Lyda sylvarum Stephens, 1835

nigricornis (Vollenhoven, 1858, Lyda)

Distribution: England, Wales

Pamphilius sylvaticus (Linnaeus, 1758)

Nomenclature:

Tenthredo sylvatica Linnaeus, 1758

Distribution: England, Scotland, Wales, Ireland

Pamphilius vafer (Linnaeus, 1767)

Nomenclature:

Tenthredo vafra Linnaeus, 1767

depressus (Schrank, 1781, Tenthredo) 
Distribution: England, Scotland, Wales, Ireland

Pamphilius varius (Serville, 1823)

Nomenclature:

Lyda varia Serville, 1823

vafer (Linnaeus, 1767): misident.

Distribution: England, Scotland, Wales, Ireland

\section{Superfamily SIRICOIDEA Billberg, 1820}

\section{Family Siricidae Billberg, 1820}

Genus Sirex Linnaeus, 1761

Sirex areolatus (Cresson, 1867)

Nomenclature:

Urocerus areolatus Cresson, 1867

Distribution: \#England

Notes: Introduced, not established.

Sirex juvencus (Linnaeus, 1758)

Nomenclature:

Ichneumon juvencus Linnaeus, 1758

nigricornis Acerbi, 1802

dubia W. F. Kirby, 1882

Distribution: England, Scotland, Wales

Notes: Sirex atricornis is considered to be a valid species (Schiff et al. 2012), which may occur in Scotland (Viitasaari and Midtgaard 1989).

Sirex noctilio Fabricius, 1793

Nomenclature:

melanocerus Thomson, 1871

Distribution: England, Scotland, Wales, Ireland 
Sirex torvus M. Harris, 1779

Nomenclature:

duplex Shuckard, 1837

cyaneus Fabricius, 1781: misident.

Distribution: England, Scotland, Wales, Ireland

Notes: A species of Central European origin, thought to be attached mainly to Abies spp. and not conspecific with the Nearctic S. cyaneus (Schiff et al. 2012).

Genus Tremex Jurine, 1807

Nomenclature:

XYLOTERUS Geoffroy, 1762

XYLOECEMATIUM Heyden, 1868

\section{Tremex columba (Linnaeus, 1763)}

Nomenclature:

Sirex columba Linnaeus, 1763

Distribution: \#England, \#Scotland

Notes: Introduced, not established.

\section{Genus Urocerus Geoffroy, 1785}

Nomenclature:

UROCERUS Geoffroy, 1762

XANTHOSIREX Semenov-Tian-Shanskij, 1921

\section{Urocerus albicornis (Fabricius, 1781)}

Nomenclature:

Sirex albicornis Fabricius, 1781

stephensii (W. F. Kirby, 1882, Sirex)

Notes: \#Introduced, not established.

Urocerus augur (Klug, 1803)

Nomenclature:

Sirex augur Klug, 1803 


\author{
bimaculata (Donovan, 1808, Sirex) \\ cedrorum (Smith, 1860, Sirex) \\ fantoma (Fabricius, 1781)
}

Distribution: England, Scotland, Ireland

Notes: Established in Ireland. Urocerus augur, U. fantoma and U. tardigradus have at times been recorded as introductions to the British Isles, but only $U$. augur is regarded as established (O'Connor et al. 1997). All were treated as valid species by Smith (1978), although $U$. tardigradus had for a long time been placed as a synonym of $U$. fantoma. Benson 1958 (ii, supplement to section a) added $U$. tardigradus to the British list with "fantoma Fabricius auctt. nec Fabricius" as its synonym and separated this in a key from "U. fantoma fantoma (= augur augur Klug)". The reasons for this treatment are not clear. No type specimens are known to exist for either $U$. fantoma or $U$. tardigradus, but Fabricius (1781) in his short original description of Sirex fantoma describes the legs as yellow, whereas $U$. augur has extensively black tibiae. European specimens previously identified as $U$. tardigradus have so far all proved to belong to $U$. fantoma (A. Taeger, pers. comm.). We revert here to treating $U$. tardigradus as a synonym of $U$. fantoma.

\title{
Urocerus californicus Norton, 1869
}

Notes: \#Introduced, not established.

\section{Urocerus fantoma (Fabricius, 1781)}

\section{Nomenclature:}

Sirex fantoma Fabricius, 1781

tardigradus (Cederhjelm, 1798, Sirex)

Notes: \#Introduced, not established.

\section{Urocerus flavicornis (Fabricius, 1781)}

\section{Nomenclature:}

Sphex flavicornis Fabricius, 1781

bizonatus (Stephens, 1835, Sirex)

\section{Distribution: \#England}

Notes: Sirex bizonatus, collected 'near London', is a synonym of the Nearctic $U$. flavicornis (Schiff et al. 2012). Presumably an introduction, not known to be established in the British Isles. 
Urocerus gigas (Linnaeus, 1758)

Nomenclature:

Ichneumon gigas Linnaeus, 1758

bizonatus (Stephens, 1835, Sirex)

taiganus Benson, 1943

Distribution: England, Scotland, Wales, Ireland

Genus Xeris Costa, 1894

Xeris spectrum (Linnaeus, 1758)

Nomenclature:

Ichneumon spectrum Linnaeus, 1758

Distribution: England, Wales, Ireland

\section{Superfamily TENTHREDINOIDEA Latreille, 1803}

Family Argidae Konow, 1890

Subfamily Arginae Konow, 1890

Genus Arge Schrank, 1802

Nomenclature:

CRYPTUS Jurine, 1801 suppressed

HYLOTOMA Latreille, 1802

Arge berberidis Schrank, 1802

Distribution: England, Wales

Notes: Added by Halstead (2004).

Arge ciliaris (Linnaeus, 1767)

Nomenclature:

Tenthredo ciliaris Linnaeus, 1767

coerulea (Klug, 1814, Hylotoma)

corusca (Zaddach, 1859, Hylotoma) 
Distribution: England, Scotland, Ireland

\section{Arge cyanocrocea (Forster, 1771)}

Nomenclature:

Tenthredo cyanocrocea Forster, 1771

coerulescens (Fabricius, 1775, Tenthredo)

coerulea (Latreille, 1805, Hylotoma)

Distribution: England, Scotland, Wales, Ireland

\section{Arge enodis (Linnaeus, 1767)}

Nomenclature:

Tenthredo enodis Linnaeus, 1767

coeruleipennis (Retzius, 1783, Tenthredo)

Distribution: England

\section{Arge expansa (Klug, 1834)}

Nomenclature:

Hylotoma expansa Klug, 1834

clavicornis (Fabricius, 1781): misident.

Distribution: Scotland, Ireland

\section{Arge fuscipes (Fallén, 1808)}

Nomenclature:

Hylotoma fuscipes Fallén, 1808

clavicornis (Fabricius, 1781): misident.

violacea (Klug, 1814, Hylotoma)

atrocoerulea (Serville, 1823, Hylotoma)

fuscinervis Lindqvist, 1974

Distribution: England, Scotland, Wales, Ireland

\section{Arge gracilicornis (Klug, 1814)}

Nomenclature:

Hylotoma gracilicornis Klug, 1814

coerulescens (Geoffroy, 1785, Tenthredo) preocc. 
incolorata (Christ, 1791, Tenthredo) nom. ob.

pilicornis (Leach, 1817, Hylotoma)

cyanella (Klug, 1834, Hylotoma)

crassa (Konow, 1884, Hylotoma)

Distribution: England, Scotland, Wales, Ireland

\section{Arge melanochra (Gmelin, 1790)}

Nomenclature:

Tenthredo melanochra Gmelin, 1790

femoralis (Klug, 1814, Hylotoma)

dimidiata (Serville, 1823, Hylotoma) preocc.

nigritarsis (Klug, 1834, Hylotoma)

bicolor (Gimmerthal, 1846, Hylotoma)

fuliginata Konow, 1907

melanochroa: misspelling

Distribution: England

\section{Arge metallica (Klug, 1834)}

Nomenclature:

Hylotoma metallica Klug, 1834

Distribution: Ireland

\section{Arge nigripes (Retzius, 1783)}

Nomenclature:

Tenthredo nigripes Retzius, 1783

enodis (Linnaeus, 1767): misident.

anglica (Leach, 1817, Hylotoma)

Distribution: England, Scotland, Wales

Arge ochropus (Gmelin, 1790)

Nomenclature:

Tenthredo ochropus Gmelin, 1790

rosincola Schrank, 1802 
rosarum (Klug, 1814, Hylotoma)

rosae (Linnaeus, 1758): misident.

ochropa: misspelling

Distribution: England

\section{Arge pagana (Panzer, 1797)}

Nomenclature:

Tenthredo pagana Panzer, 1797

?stephensii (Leach, 1817, Hylotoma)

Distribution: England, Wales

Notes: Arge stephensii differs very markedly, in the extreme development of pale body markings, from all known W. Palaearctic populations of $A$. pagana. Traditionally, $A$. stephensii has been regarded as an endemic British subspecies of $A$. pagana. Further studies [here a DNA analysis might offer good clues] are definitely required. The possibility cannot be excluded that $A$. stephensii is a separate species originally introduced from the E. Palaearctic or Oriental Regions, where Arge is both extremely species-rich and poorly investigated.

\section{Arge rustica (Linnaeus, 1758)}

\section{Nomenclature:}

Tenthredo rustica Linnaeus, 1758

atrata (Forster, 1771, Tenthredo)

segmentaria (Panzer, 1803, Cryptus)

klugii (Leach, 1817, Hylotoma)

leachii (Stephens, 1835, Hylotoma)

Distribution: England

\section{Arge ustulata (Linnaeus, 1758)}

Nomenclature:

Tenthredo ustulata Linnaeus, 1758

Distribution: England, Scotland, Wales, Ireland 
Subfamily Sterictiphorinae Rohwer, 1911

Genus Aprosthema Konow, 1899

Nomenclature:

COPIDOCEROS Forsius, 1921

Aprosthema fusicorne (Thomson, 1871)

Nomenclature:

Schizocera fusicornis Thomson, 1871

Distribution: England

Notes: Added by Vikberg (2004).

Aprosthema melanurum (Klug, 1814)

Nomenclature:

friesei (Konow, 1895, Schizocera)

alfkeni (Konow, 1895, Schizocera)

melanura: misspelling

Distribution: England

Aprosthema tardum (Klug, 1814)

Nomenclature:

Hylotoma tarda Klug, 1814

Distribution: England

Notes: Added by Vikberg (2004).

Genus Sterictiphora Billberg, 1820

Nomenclature:

SCHIZOCERUS Berthold, 1827

SCHIZOCERA Lepeletier \& Serville, 1828

CYPHONA Dahlbom, 1835

SCHIZOCEROS Konow, 1899

STERICTOPHORA Benson, 1951: misspelling 
Sterictiphora angelicae (Panzer, 1799)

Nomenclature:

$$
\begin{aligned}
& \text { villersii (Leach, 1817, Cryptus) } \\
& \text { melanocephala (Fabricius, 1798): misident. } \\
& \text { furcata (Villers, 1789): misident. }
\end{aligned}
$$

Distribution: England, Wales

Sterictiphora geminata (Gmelin, 1790)

Nomenclature:

Tenthredo geminata Gmelin, 1790

pallipes (Leach, 1817, Cryptus)

Distribution: England, Scotland, Wales, Ireland

Family Blasticotomidae Thomson, 1871

Genus Blasticotoma Klug, 1834

Blasticotoma filiceti Klug, 1834

Distribution: England, Wales, Ireland

Family Cimbicidae Curtis, 1825

Subfamily Abiinae Benson, 1951

Notes: Benson (1951) treated Abia and Zaraea as belonging to two different tribes. The differences between these certainly do not warrant this (Lorenz and Kraus 1957, Taeger 1998).

Genus Abia Leach, 1817

Nomenclature:

ZARAEA Leach, 1817

AENOABIA Kangas, 1946

AUROABIA Kangas, 1946 
Abia aenea (Klug, 1820)

Nomenclature:

bifida (Thomson, 1871, Abia)

bigens (Kangas, 1946, Abia)

Ionicerae (Linnaeus, 1758): Enslin, 1917 misident.

Distribution: England

Abia candens Konow, 1887

Distribution: England, Scotland, Wales, Ireland

Abia fasciata (Linnaeus, 1758)

Nomenclature:

Tenthredo fasciata Linnaeus, 1758

Distribution: England, Scotland, Wales, Ireland

Abia lonicerae (Linnaeus, 1758)

Nomenclature:

nigricornis Leach, 1817

Distribution: England, Wales, Ireland

Abia sericea (Linnaeus, 1767)

Nomenclature:

Tenthredo sericea Linnaeus, 1767

Distribution: England, Scotland, Wales, Ireland

Subfamily Cimbicinae Curtis, 1825

Tribe Cimbicini Curtis, 1825

Genus Cimbex Olivier, 1790

Nomenclature:

CRABRO Geoffroy, 1762 suppressed

CLAVELLARIUS Olivier, 1789 suppressed

CLAVELLARIA Lamarck, 1801 
PALAEOCIMBEX Semenov Tian-Shanskij, 1935

ALLOCIMBEX Zirngiebl, 1953

\section{Cimbex connatus (Schrank, 1776)}

Nomenclature:

Tenthredo connata Schrank, 1776

Distribution: England, Wales, Ireland

\section{Cimbex femoratus (Linnaeus, 1758)}

Nomenclature:

Tenthredo femorata Linnaeus, 1758

europaea Leach, 1817

sylvarum (Fabricius, 1793, Tenthredo)

varians Leach, 1817

Distribution: England, Scotland, Wales, Ireland

\section{Cimbex luteus (Linnaeus, 1758)}

Nomenclature:

Tenthredo lutea Linnaeus, 1758

griffinii Leach, 1817

annulata (Geoffroy, 1785): Leach, 1817 misident.

Distribution: England, Scotland, Wales

\section{Cimbex quadrimaculatus (Müller, 1766)}

\section{Nomenclature:}

Tenthredo quadrimaculata Müller, 1766

humeralis (Geoffroy, 1785, Crabro)

axillaris (Panzer, 1801, Tenthredo)

Distribution: †England

Notes: Extinct in Britain; presence in British Isles based on one female (NHM) said to have been collected near Salisbury (Leach 1817, Stephens 1835). 
Tribe Trichiosomini Benson, 1951

Genus Pseudoclavellaria Schulz, 1906

Nomenclature:

CLAVELLARIA Lamarck, 1801: misident.

Pseudoclavellaria amerinae (Linnaeus, 1758)

Nomenclature:

Tenthredo amerinae Linnaeus, 1758

marginata (Linnaeus, 1767, Tenthredo)

Distribution: †England

Notes: Extinct in Britain; reported as having been collected near Windsor (Leach 1817, Stephens 1835).

Genus Trichiosoma Leach, 1817

Nomenclature:

ASITRICHIOSOMA Malaise, 1937

Notes: Taxonomy follows Viitasaari (1985), Viitasaari (1989).

Trichiosoma laterale Leach, 1817

Nomenclature:

?marginale Leach, 1817

latreillei Leach, 1817

Distribution: England, Scotland, Ireland

Trichiosoma lucorum (Linnaeus, 1758)

Nomenclature:

Tenthredo lucorum Linnaeus, 1758

Distribution: England, Scotland, Wales, Ireland 
Trichiosoma pusillum Stephens, 1835

Nomenclature:

nigripes Gussakovskij, 1947

Distribution: England

Trichiosoma scalesii Leach, 1817

Nomenclature:

unidentatum Leach, 1817

silvaticum Leach, 1817

biverrucatum Stephens, 1835

betuleti (Klug, 1834): Cameron, 1875 misident.

Distribution: England, Scotland

Trichiosoma sorbi Hartig, 1840

Nomenclature:

scalesii Leach, 1817: Cameron, 1885 misident.

Distribution: England, Scotland, Wales, Ireland

Trichiosoma tibiale Stephens, 1835

Nomenclature:

crataegi (Zaddach, 1863, Cimbex)

Distribution: England, Scotland, Wales, Ireland

Trichiosoma vitellina (Linnaeus, 1760)

Nomenclature:

Tenthredo vitellina Linnaeus, 1760

boreale Gussakovskij, 1947

vitellinae: misspelling

Distribution: England, Scotland, Ireland

Subfamily Corynidinae Benson, 1938

Nomenclature:

CORYNINAE preocc. 


\section{Genus Corynis Thunberg, 1789}

Nomenclature:

AMASIS Leach, 1817

Corynis crassicornis (Rossi, 1790)

Nomenclature:

Tenthredo crassicornis Rossi, 1790

laeta (Fabricius, 1798, Tenthredo)

Distribution: ?England

Notes: Said to have been collected near Bristol (Leach 1817, Stephens 1835).

Corynis obscura (Fabricius, 1775)

Nomenclature:

Tenthredo obscura Fabricius, 1775

Distribution: ?England

Notes: Said to have been collected in Lincolnshire (Leach 1817, Stephens 1835).

Family Diprionidae Rohwer, 1910

Subfamily Diprioninae Rohwer, 1910

Genus Diprion Schrank, 1802

Nomenclature:

PTERONUS Jurine, 1801 suppressed

LOPHYRUS Latreille, 1802 suppressed

Diprion pini (Linnaeus, 1758)

Nomenclature:

Tenthredo pini Linnaeus, 1758

Distribution: England, Scotland, Wales, Ireland

Diprion similis (Hartig, 1836)

Nomenclature:

Lophyrus similis Hartig, 1836 
eremita (Thomson, 1871, Lophyrus)

Distribution: England, Scotland, Wales

Genus Gilpinia Benson, 1939

Gilpinia frutetorum (Fabricius, 1793)

Nomenclature:

Tenthredo frutetorum Fabricius, 1793

thomsoni (Konow, 1884, Lophyrus)

Lophyrus variegatus Hartig, 1834: Cameron, 1890 misident.

Distribution: Scotland

Gilpinia hercyniae (Hartig, 1837)

Nomenclature:

Lophyrus hercyniae Hartig, 1837

polytoma (Hartig, 1834): misident.

Distribution: England, Wales

Gilpinia pallida (Klug, 1812)

Nomenclature:

Lophyrus pallidus Klug, 1812

Lophyrus virens (Klug, 1812): Cameron, 1885 misident.

Lophyrus dorsatus (Fabricius, 1781): Cameron, 1890 misident.

Distribution: England, Scotland

\section{Gilpinia virens (Klug, 1812)}

Nomenclature:

Lophyrus virens Klug, 1812

Distribution: England 
Genus Microdiprion Enslin, 1917

Microdiprion pallipes (Fallén, 1808)

Nomenclature:

Hylotoma pallipes Fallén, 1808

Distribution: England, Scotland, Wales

Genus Neodiprion Rohwer, 1918

Neodiprion sertifer (Geoffroy, 1785)

Nomenclature:

Tenthredo sertifera Geoffroy, 1785

rufa (Retzius, 1783): Latreille, 1807 misident.

Distribution: England, Scotland, Wales, Ireland

Subfamily Monocteninae Benson, 1945

Genus Monoctenus Dahlbom, 1835

Monoctenus juniperi (Linnaeus, 1758)

Nomenclature:

Tenthredo juniperi Linnaeus, 1758

Distribution: Scotland, Ireland

Family Heptamelidae Benson, 1938

Notes: Previously treated as a tribe of Selandriinae, raised to family rank by Malm and Nyman (2014) as the inclusion of Heptamelus and Pseudoheptamelus in the Tenthredinidae rendered the latter paraphyletic with respect to Cimbicidae and Diprionidae.

Heptamelus dahlbomi (Thomson, 1870)

Nomenclature:

Caenoneura dahlbomi Thomson, 1870

ochroleucus: misident.

Distribution: England, Scotland, Ireland 
Notes: Added by Vikberg and Liston (2009).

Heptamelus ochroleucus (Stephens, 1835)

Nomenclature:

Melicerta ochroleucus Stephens, 1835

Distribution: England, Scotland, Wales, Ireland

Family Tenthredinidae Latreille, 1802

Notes: Unplaced species of Tenthredinidae:

Selandria ornata Newman, 1838

Subfamily Allantinae Rohwer, 1911

Tribe Allantini Rohwer, 1911

Genus Allantus Panzer, 1801

Nomenclature:

EMPHYTUS Klug, 1815

Notes: According to Stephens (1835) (p.90), who gives a recognisable description of A. didymus (Klug, 1818), this species was “..rare: taken at Birch wood in June." It is not included in the list of British species because no authenticated material has been located. A specimen without locality data in the Stephens Collection (NHM) is $A$. melanarius (det. G. Knight).

\section{Allantus basalis (Klug, 1818)}

Nomenclature:

Tenthredo basalis Klug, 1818

?basalis ssp. caledonicus (Benson, 1945, Emphytus)

Distribution: England, Scotland

Notes: Allantus basalis caledonicus may represent a separate species (Blank and Taeger 1998) from Scottish specimens recorded as $A$. basalis ssp. basalis by Liston (1985) and similar specimens from northern England. 
Allantus calceatus (Klug, 1818)

Nomenclature:

Tenthredo calceata Klug, 1818

Distribution: England, Scotland, Wales, Ireland

Allantus cinctus (Linnaeus, 1758)

Nomenclature:

Tenthredo cincta Linnaeus, 1758

neglectus (Zaddach, 1859, Emphytus)

cinctipes (Norton, 1867, Emphytus)

Distribution: England, Scotland, Wales, Ireland

Allantus cingulatus (Scopoli, 1763)

Nomenclature:

Tenthredo cingulata Scopoli, 1763

Distribution: England, Wales, Ireland

Allantus coryli (Stritt, 1937)

Nomenclature:

Emphytus coryli Stritt, 1937

Distribution: England

Allantus laticinctus (Serville, 1823)

Nomenclature:

Dolerus laticinctus Serville, 1823

balteatus (Klug, 1818, Tenthredo) preocc.

Distribution: Wales

Notes: Added by Knight (2006).

Allantus melanarius (Klug, 1818)

Nomenclature:

Tenthredo melanaria Klug, 1818

Distribution: England 
Allantus rufocinctus (Retzius, 1783)

Nomenclature:

Tenthredo rufocincta Retzius, 1783

Distribution: England, Scotland, Wales, Ireland

\section{Allantus togatus (Panzer, 1801)}

Nomenclature:

Tenthredo togata Panzer, 1801

Distribution: England, Scotland, Wales

\section{Allantus truncatus (Klug, 1818)}

Nomenclature:

Tenthredo truncata Klug, 1818

cingillum (Klug, 1818): Morice, 1909 misident.

melanarius (Klug, 1818): Morice, 1909 misident.

Distribution: England, Scotland, Wales

Genus Apethymus Benson, 1939

Apethymus filiformis (Klug, 1818)

Nomenclature:

Tenthredo filiformis Klug, 1818

serotinus (Klug, 1818, Tenthredo) preocc.

abdominalis (Serville, 1823, Dolerus)

autumnalis (Forsius, 1933, Emphytus)

Distribution: England, Scotland, Wales, Ireland

\section{Apethymus serotinus (O. F. Müller, 1776)}

Nomenclature:

Tenthredo serotina Müller, 1776

braccatus (Gmelin, 1790, Tenthredo)

tibialis (Panzer, 1799, Tenthredo) preocc.

panzeri (Kirby, 1882, Emphytus) 
Distribution: England, Scotland, Wales, Ireland

Genus Taxonus Hartig, 1837

Taxonus agrorum (Fallén, 1808)

Nomenclature:

Tenthredo agrorum Fallén, 1808

Distribution: England, Scotland

Tribe Caliroini Benson, 1938

Genus Caliroa Costa, 1859

Nomenclature:

ERIOCAMPOIDES Konow, 1890

\section{Caliroa annulipes (Klug, 1816)}

Nomenclature:

Tenthredo annulipes Klug, 1816

atra (Stephens, 1835, Selandria)

Distribution: England, Scotland, Wales, Ireland

Caliroa cerasi (Linnaeus, 1758)

Nomenclature:

Tenthredo cerasi Linnaeus, 1758

limacina (Retzius, 1783, Tenthredo)

adumbrata (Klug, 1816, Tenthredo)

antipoda (W. F. Kirby, 1881, Monostegia)

Distribution: England, Scotland, Wales, Ireland

Caliroa cinxia (Klug, 1816)

Nomenclature:

Tenthredo cinxia Klug, 1816

Distribution: England 
Caliroa tremulae Chevin, 1974

Nomenclature:

varipes (Klug, 1816): misident.

Distribution: England

Notes: Added by Liston (1993).

\section{Caliroa varipes (Klug, 1816)}

Nomenclature:

Tenthredo varipes Klug, 1816

Distribution: England, Wales, Ireland

Genus Endelomyia Ashmead, 1898

Endelomyia aethiops (Gmelin, 1790)

Nomenclature:

Tenthredo aethiops Gmelin, 1790

soror (Vollenhoven, 1869, Selandria)

testaceipes (Cameron, 1874, Eriocampa)

caninae (Cameron, 1878, Eriocampa)

nigricolle (Cameron, 1882, Poecilosoma)

Distribution: England, Scotland, Wales, Ireland

Tribe Empriini Rohwer, 1911

Genus Ametastegia Costa, 1882

Nomenclature:

PROTEMPHYTUS Rohwer, 1909

PROTOEMPHYTUS: misspelling

Ametastegia albipes (Thomson, 1871)

Nomenclature:

Taxonus albipes Thomson, 1871

fletcheri (Cameron, 1878, Taxonus)

Emphytus nigricans (Klug, 1818): Stephens, 1835 misident. 
Distribution: England, Wales, Ireland

Ametastegia carpini (Hartig, 1837)

Nomenclature:

Emphytus carpini Hartig, 1837

glottianus (Cameron, 1874, Taxonus)

Distribution: England, Scotland, Wales, Ireland

Notes: Taxonus glottianus was justifiably synonymised with Ametastegia tenera by Konow (1899), because the original description strongly indicates the latter species, but after examination of the type Benson (1952) regarded T. glottianus as a junior synonym of $A$. carpini. We follow Benson's decision on the status of Cameron's name.

Ametastegia equiseti (Fallén, 1808)

Nomenclature:

Tenthredo equiseti Fallén, 1808

coxalis (Hartig, 1837, Tenthredo)

Distribution: England, Scotland, Wales, Ireland

Ametastegia glabrata (Fallén, 1808)

Nomenclature:

Tenthredo glabrata Fallén, 1808

Distribution: England, Scotland, Wales, Ireland

Ametastegia pallipes (Spinola, 1808)

Nomenclature:

Tenthredo pallipes Spinola, 1808

grossulariae (Klug, 1818, Tenthredo)

Distribution: England, Scotland, Wales, Ireland

Ametastegia perla (Klug, 1818)

Nomenclature:

Tenthredo perla Klug, 1815

Distribution: England, Scotland, Ireland 
Ametastegia tenera (Fallén, 1808)

Nomenclature:

Tenthredo tenera Fallén, 1808

patellata (Klug, 1818, Tenthredo)

tener: misspelling

Distribution: England, Scotland, Wales, Ireland

Genus Empria Lepeletier \& Serville, 1828

Nomenclature:

POECILOSTOMA Dahlbom, 1835

POECILOSOMA Thomson, 1870 preocc.

PARATAXONUS MacGillivray, 1908

LEUCEMPRIA Enslin, 1913

TRIEMPRIA Enslin, 1914

Notes: Unplaced species of Empria:

[signata (Newman, 1838, Selandria)]

Empria alector Benson, 1938

Distribution: England, Scotland, Wales, Ireland

Empria alpina Benson, 1938

Nomenclature:

gussakovskii Dovnar-Zapolskij, 1929: Zhelochovtsev, 1988 misident.

Distribution: Scotland, Ireland

Empria basalis Lindqvist, 1968

Distribution: England, Scotland

Notes: Added by Knight (2009).

Empria candidata (Fallén, 1808)

Nomenclature:

Tenthredo candidata Fallén, 1808

repanda (Klug, 1816, Tenthredo) 
Distribution: England, Scotland, Wales

Empria excisa (Thomson, 1871)

Nomenclature:

Poecilosoma excisa Thomson, 1871

Distribution: England, Scotland, Wales, Ireland

Empria fletcheri (Cameron, 1878)

Nomenclature:

Poecilosoma fletcheri Cameron, 1878

obtusa (Klug, 1817): Cameron, 1874 misident.

Distribution: Scotland

Empria immersa (Klug, 1818)

Nomenclature:

Tenthredo immersa Klug, 1818

tirolensis Enslin, 1914

Distribution: England, Scotland, Wales, Ireland

Empria liturata (Gmelin, 1790)

Nomenclature:

Tenthredo liturata Gmelin, 1790

Distribution: England, Scotland, Wales, Ireland

Empria longicornis (Thomson, 1871)

Nomenclature:

Poecilosoma longicornis Thomson, 1871

rubi Kontuniemi, 1951

Distribution: England, Scotland, Wales, Ireland

Empria minuta Lindqvist, 1968

Distribution: Scotland 
Notes: Added by Knight \& Liston (in prep.).

Empria pallimacula (Serville, 1823)

Nomenclature:

Dolerus pallimacula Serville, 1823

baltica Conde, 1937

Distribution: England, Scotland, Wales, Ireland

\section{Empria parvula (Konow, 1892)}

Nomenclature:

Poecilosoma parvula Konow, 1892

Distribution: England, Scotland

\section{Empria pumila (Konow, 1896)}

Nomenclature:

Poecilosoma pumila Konow, 1896

Distribution: England, Scotland, Wales, Ireland

Notes: Empria pumiloides Lindqvist, 1968 is distinguished from E. pumila by Heidemaa and Viitasaari (1999). Although Scottish and Welsh specimens checked by Knight and Liston are all E. pumila, the presence of E. pumiloides in the British Isles cannot be ruled out.

\section{Empria sexpunctata (Serville, 1823)}

\section{Nomenclature:}

Tenthredo sexpunctata Serville, 1823

klugii (Stephens, 1835, Selandria)

carbonaria (Konow, 1884, Poecilosoma)

klugi: misspelling

guttata (Fallén, 1808): Cameron, 1875 misident.

Distribution: England, Scotland, Wales, Ireland

\section{Empria tridens (Konow, 1896)}

Nomenclature:

Poecilosoma tridens Konow, 1896 
Distribution: England, Scotland, Wales, Ireland

Genus Harpiphorus Hartig, 1837

Nomenclature:

ASTICTA Newman, 1838 preocc.

Harpiphorus lepidus (Klug, 1818)

Nomenclature:

Tenthredo lepida Klug, 1818

ianthe (Newman, 1837, Fenusa)

Distribution: England, Wales, Ireland

Genus Monostegia Costa, 1859

Nomenclature:

NEMATOCEROS Konow, 1896

Monostegia abdominalis (Fabricius, 1798)

Nomenclature:

Tenthredo abdominalis Fabricius, 1798

luteola (Klug, 1816, Tenthredo)

Distribution: England, Wales, Ireland

Genus Monsoma MacGillivray, 1908

Nomenclature:

MONOSOMA Viereck, 1910

Monsoma pulveratum (Retzius, 1783)

Nomenclature:

Tenthredo pulverata Retzius, 1783

pulverata: misspelling

Distribution: England, Scotland, Wales, Ireland 
Tribe Eriocampini Rohwer, 1911

Genus Eriocampa Hartig, 1837

Nomenclature:

BRACHYOCAMPA Zirngiebl, 1956

Eriocampa ovata (Linnaeus, 1760)

Nomenclature:

Tenthredo ovata Linnaeus, 1760

Distribution: England, Scotland, Wales, Ireland

\section{Subfamily Athaliinae Rohwer, 1911}

Notes: Raised to subfamily rank by Malm and Nyman (2014).

Genus Athalia Leach, 1817

Nomenclature:

DENTATHALIA Benson, 1931

Athalia ancilla Serville, 1823

Nomenclature:

glabricollis Thomson, 1870

Distribution: England, Scotland, Wales

Athalia bicolor Serville, 1823

Nomenclature:

annulata (Fabricius, 1787, Tenthredo) preocc.

richardi Serville, 1823

Distribution: England, Wales

Athalia circularis (Klug, 1815)

Nomenclature:

Tenthredo circularis Klug, 1815

lineolata Serville, 1823

?cordatoides Kontuniemi, 1951 
Distribution: England, Scotland, Wales, Ireland

Notes: There are grounds for suspecting that this consists of a group of species, with more narrowly defined host plant spectra than presently accepted.

Athalia cordata Serville, 1823

Distribution: England, Scotland, Wales, Ireland

\section{Athalia cornubiae Benson, 1931}

Distribution: England

\section{Athalia liberta (Klug, 1815)}

Nomenclature:

Tenthredo liberta Klug, 1815

Distribution: England, Scotland, Wales, Ireland

\section{Athalia lugens (Klug, 1815)}

Nomenclature:

Tenthredo lugens Klug, 1815

Distribution: England, Scotland, Wales, Ireland

\section{Athalia rosae (Linnaeus, 1758)}

Nomenclature:

Tenthredo rosae Linnaeus, 1758

colibri (Christ, 1791, Tenthredo)

spinarum (Fabricius, 1793, Tenthredo)

centifoliae (Panzer, 1797, Tenthredo)

Distribution: England, Scotland, Wales

\section{Athalia scutellariae Cameron, 1880}

\section{Nomenclature:}

$$
\text { galericulatae (Kontuniemi, 1951, Dentathalia) }
$$

Distribution: England, Scotland, Wales, Ireland 
Subfamily Blennocampinae Konow, 1890

Tribe Blennocampini Konow, 1890

Genus Ardis Konow, 1886

Ardis pallipes (Serville, 1823)

Nomenclature:

Dolerus pallipes Serville, 1823

brunniventris (Hartig, 1837, Tenthredo)

bipunctata (Klug, 1817, Tenthredo) preocc.

Distribution: England, Scotland, Wales, Ireland

Ardis sulcata (Cameron, 1882)

Nomenclature:

Blennocampa sulcata Cameron, 1882

Distribution: England, Wales

Genus Blennocampa Hartig, 1837

Blennocampa phyllocolpa Viitasaari \& Vikberg, 1985

Nomenclature:

pusilla (Klug, 1816, Tenthredo) preocc.

Distribution: England, Scotland, Wales, Ireland

Genus Cladardis Benson, 1952

Cladardis elongatula (Klug, 1817)

Nomenclature:

Tenthredo elongatula Klug, 1817

sericans (Hartig, 1837): Cameron, 1882 misident.

Distribution: ?England

Notes: Presence in the British Isles uncertain: see Gibbs (2006). 


\section{Genus Claremontia Rohwer, 1909}

Nomenclature:

PSEUDOBLENNOCAMPA Malaise, 1935

\section{Claremontia alchemillae (Cameron, 1877)}

Nomenclature:

Blennocampa alchemillae Cameron, 1877

Distribution: England, Scotland, Wales, Ireland

Notes: The taxonomy of this species, C. tenuicornis (Klug, 1816) and C. uncta (Klug, 1816) has not yet been fully resolved (Liston et al. 2006).

\section{Claremontia alternipes (Klug, 1816)}

Nomenclature:

Tenthredo alternipes Klug, 1816

intermedia (Kriechbaumer, 1884, Blennocampa)

tergestina (Kriechbaumer, 1888, Blennocampa)

cinereipes (Klug, 1816): Thomson, 1870 misident.

\section{Claremontia brevicornis (Brischke, 1883)}

Nomenclature:

Blennocampa brevicornis Brischke, 1883

confusa (Konow, 1886, Blennocampa)

geniculata (Hartig, 1837): Stephens, 1835 misident.

alternipes (Klug, 1816): misident.

Distribution: England, Scotland, Wales, Ireland

\section{Claremontia puncticeps (Konow, 1886)}

Nomenclature:

Blennocampa puncticeps Konow, 1886

Distribution: England, Wales 


\section{Claremontia tenuicornis (Klug, 1816)}

Nomenclature:

Tenthredo tenuicornis Klug, 1816

?tibialis (Stephens, 1835, Selandria)

spiraeae (Brischke, 1883, Blennocampa)

geniculata (Hartig, 1837): Enslin, 1918 misident.

Distribution: England, Scotland, Wales

Notes: Many previous records of this species in the British Isles seem to refer to $C$. alchemillae and $C$. uncta.

\section{Claremontia uncta (Klug, 1816)}

\section{Nomenclature:}

Tenthredo uncta Klug, 1816

humeralis (Vollenhoven, 1869, Blennocampa)

Distribution: England, Scotland, Ireland

Notes: Added by Koch (1988).

See above under C. alchemillae / C. tenuicornis.

\section{Claremontia waldheimii (Gimmerthal, 1847)}

Nomenclature:

Tenthredo waldheimii Gimmerthal, 1847

subcana (Zaddach, 1859, Selandria)

subserrata (Thomson, 1870, Blennocampa)

Distribution: England, Scotland, Wales

\section{Genus Monardis Hartig, 1837}

Nomenclature:

Tenthredo plana Klug, 1817

\section{Monardis plana (Klug, 1817)}

Nomenclature:

Tenthredo plana Klug, 1817

rosarum (Brischke, 1883, Blennocampa) 
Distribution: Wales

Notes: Added by Gibbs (2006).

Genus Monophadnoides Ashmead, 1898

Nomenclature:

PSEUDOMONOPHADNUS Malaise, 1935

Monophadnoides rubi (T.W. Harris, 1845)

Nomenclature:

Selandria rubi Harris, 1845

geniculata (Hartig, 1837, Tenthredo) preocc.

Distribution: England, Scotland, Wales, Ireland

Monophadnoides ruficruris (Brullé, 1832)

Nomenclature:

Selandria ruficruris Brullé, 1832

Distribution: England, Scotland, Wales

\section{Genus Pareophora Konow, 1886}

Nomenclature:

Tenthredo pruni Linnaeus, 1758

Pareophora pruni (Linnaeus, 1758)

Nomenclature:

Tenthredo pruni Linnaeus, 1758

nigripes (Klug, 1816, Tenthredo) preocc.

Distribution: England, Ireland

Genus Periclista Konow, 1886

Periclista albida (Klug, 1816)

Nomenclature:

Tenthredo albida Klug, 1816

melanocephala (Fabricius, 1798, Tenthredo) preocc. 
versicolor (Newman, 1837, Selandria)

Distribution: England, Scotland, Wales, Ireland

Periclista lineolata (Klug, 1816)

Nomenclature:

Tenthredo lineolata Klug, 1816

Distribution: England, Scotland

Periclista pubescens (Zaddach, 1859)

Nomenclature:

Selandria pubescens Zaddach, 1859

Distribution: England, Scotland, Wales

Tribe Fenusini MacGillivray, 1906

Genus Fenella Westwood, 1840

Nomenclature:

PARAPHYLLOTOMA Forsius, 1930

Fenella monilicornis (Dahlbom, 1835)

Nomenclature:

Phyllotoma monilicornis Dahlbom, 1835

famosa Benson, 1950

minuta (Dahlbom, 1835): misident.

Distribution: Scotland

Fenella nigrita Westwood, 1840

Nomenclature:

tormentillae (Healy, 1868, Phyllotoma)

?agrimoniae Brischke, 1888

pygmaea (Klug, 1816): Healy, 1869 misident.

Distribution: England, Scotland, Wales, Ireland 
Notes: Benson (1952) noted that biological differences possibly indicate the presence of two taxa under this name in the British Isles. This problem has still not been investigated.

\section{Genus Fenusa Leach, 1817}

Nomenclature:

KALIOSYSPHINGA Tischbein, 1846

APHADNURUS Costa, 1859

PHAENUSA Cameron, 1875

CALIOSYSPHINGA Konow, 1905

\section{Fenusa dohrnii (Tischbein, 1846)}

Nomenclature:

Kaliosysphinga dohrnii Tischbein, 1846

melanopoda (Cameron, 1876, Phaenusa)

westwoodi (Cameron, 1882, Fenella)

Distribution: England, Scotland, Wales, Ireland

\section{Fenusa pumila Leach, 1817}

\section{Nomenclature:}

pumila (Klug, 1818, Tenthredo) preocc.

pusilla (Lepeletier, 1823): misident.

fuliginosa Healy, 1869

minima Brischke, 1883

pygmaea (Klug, 1816): Zetterstedt, 1838 misident.

Distribution: England, Scotland, Wales, Ireland

Genus Fenusella Enslin, 1914

Nomenclature:

MESSA Leach, 1817: misident.

Fenusella glaucopis (Konow, 1907)

Nomenclature:

Fenusa glaucopis Konow, 1907 
Distribution: England, Scotland, Wales

Fenusella hortulana (Klug, 1818)

Nomenclature:

Tenthredo hortulana Klug, 1818

soenderupi Hering, 1935

Distribution: England, Ireland

Fenusella nana (Klug, 1816)

Nomenclature:

Tenthredo nana Klug, 1816

mellita (Newman, 1870, Phyllotoma)

quercus (Cameron, 1885, Fenusa)

laeta (Enslin, 1918, Scolioneura)

Distribution: England, Scotland, Wales, Ireland

Genus Heterarthrus Stephens, 1835

Nomenclature:

PHYLLOTOMA Fallén, 1829 preocc.

DECATRIA Stephens, 1835

DRUIDA Newman, 1838

HETERARTHUS Cameron, 1882

Notes: Unplaced species of Heterarthrus:

[fumipennis (Cameron, 1888, Phyllotoma)]

Heterarthrus aceris (Kaltenbach, 1856)

Nomenclature:

Phyllotoma aceris Kaltenbach, 1856

Distribution: England, Scotland, Wales, Ireland

Heterarthrus cuneifrons Altenhofer \& Zombori, 1987

Distribution: England

Notes: Added by Liston and Blank (2006). 
Heterarthrus microcephalus (Klug, 1818)

Nomenclature:

Tenthredo microcephala Klug, 1818

melanopyga (Klug, 1818): Healy, 1868 misident.

Distribution: England, Scotland, Ireland

Heterarthrus nemoratus (Fallén, 1808)

Nomenclature:

Hylotoma nemorata Fallén, 1808

parviceps (Newman, 1837, Fenusa)

tenellus (Zaddach, 1859, Phyllotoma)

mathesoni (MacGillivray, 1909, Phlebotrophia)

mathewsoni: misspelling

Distribution: England, Scotland, Wales, Ireland

Heterarthrus ochropoda (Klug, 1818)

Nomenclature:

Tenthredo ochropoda Klug, 1818

fuscipennis (Stephens, 1835, Decatria)

pinguis (Vollenhoven, 1869, Phyllotoma)

maxima (Strobl, 1896, Phyllotoma)

ochropodus: misspelling

Distribution: England, Scotland, Wales

Heterarthrus vagans (Fallén, 1808)

Nomenclature:

Hylotoma vagans Fallén, 1808

melanopygus (Klug, 1818, Tenthredo)

amaurus (Klug, 1818, Tenthredo)

kamtchaticus (Malaise, 1931, Phyllotoma)

Distribution: England, Scotland, Wales, Ireland 


\section{Heterarthrus wuestneii (Konow, 1905)}

Nomenclature:

Phyllotoma wuestneii Konow, 1905

aceris (McLachlan, 1867, Phyllotoma) preocc.

healyi Altenhofer \& Zombori, 1987

Distribution: England

Notes: Added by Altenhofer and Zombori (1987).

Phyllotoma aceris was described on the basis of specimens "bred by Mr Healy from larvae which make great blotches in the leaves of Acer campestre, and occasionally in Acer pseudo-platanus" McLachlan (1867). According to present knowledge on distribution and hostplants, the syntype series probably consisted of more than one species. The description is not sufficiently detailed to determine the identity of the specimens which McLachlan examined. No type material has been located, as already stated by Altenhofer and Zombori (1987). Nevertheless, Altenhofer and Zombori (1987) chose to regard $P$. aceris McLachlan as being conspecific with a species which was reared from $A$. campestre and proposed for it the new name Heterarthrus healyi. $H$. healyi was subsequently found to be synonymous with $H$. wuestneii (Konow, 1905) (Blank et al. 2001).

\section{Genus Kaliofenusa MacGillivray, 1910}

Notes: Unplaced species of Kaliofenusa:

[pusilla (Serville, 1823, Dolerus)]

\section{Kaliofenusa altenhoferi Liston, 1993}

\section{Nomenclature:}

carpinifoliae Liston, 1993

Distribution: England, Scotland

Notes: Added by Liston (1994). Synonymy with K. carpinifoliae according to Liston (1996). Dolerus pusillus Serville cannot at present be placed as conspecific with K. ulmi or K. altenhoferi, and must be treated as an unplaced species (Blank et al. 2001).

\section{Kaliofenusa ulmi (Sundevall, 1847)}

\section{Nomenclature:}

Fenusa ulmi Sundevall, 1847

intermedia (Thomson, 1871, Fenusa) 
alsia (MacGillivray, 1923, Messa)

Distribution: England, Scotland, Wales, Ireland

Notes: Recorded in Ireland by Knight (2004) as K. pusilla.

Genus Metallus Forbes, 1885

Nomenclature:

ENTODECTA Konow, 1886

POLYBATES MacGillivray, 1909

\section{Metallus albipes (Cameron, 1875)}

Nomenclature:

Phaenusa albipes Cameron, 1875

tenuicornis (Hellén, 1935, Entodecta)

Distribution: England, Scotland

Metallus lanceolatus (Thomson, 1870)

Nomenclature:

Blennocampa lanceolata Thomson, 1870

gei (Brischke, 1883, Fenusa)

decolor (Konow, 1886, Entodecta)

bensoni Smith, 1971

Distribution: England, Scotland, Wales

\section{Metallus pumilus (Klug, 1816)}

Nomenclature:

Tenthredo pumila Klug, 1816

pumilio (Hartig, 1837, Emphytus)

rubi (Boie, 1848, Fenusa)

Distribution: England, Scotland, Ireland 
Genus Parna Benson, 1936

Parna apicalis (Brischke, 1888)

Nomenclature:

Blennocampa apicalis Brischke, 1888

reseri Liston, 1993

Distribution: England, Scotland, Wales

Notes: Added by Edmunds et al. (2007).

Parna tenella (Klug, 1816)

Nomenclature:

Tenthredo tenella Klug, 1816

tiliae (Kaltenbach, 1874, Blennocampa)

Distribution: England, Scotland, Wales

Genus Profenusa MacGillivray, 1914

Profenusa pygmaea (Klug, 1816)

Nomenclature:

Tenthredo pygmaea Klug, 1816

Distribution: England, Scotland, Wales, Ireland

Profenusa thomsoni (Konow, 1886)

Nomenclature:

Fenusa thomsoni Konow, 1886

alumna (MacGillivray, 1923): misident.

Distribution: England

Genus Scolioneura Konow, 1890

Scolioneura betuleti (Klug, 1816)

Nomenclature:

Tenthredo betuleti Klug, 1816 
nigricans (Klug, 1818, Tenthredo) preocc.

betulae (Zaddach, 1859, Fenusa)

vicina Konow, 1894

Distribution: England, Scotland, Ireland

Notes: Synonymy of S. vicina with S. betuleti follows Leppänen et al. (2012).

Tribe Phymatocerini Rohwer, 1911

Nomenclature:

TOMOSTETHINI Benson, 1938

Genus Eutomostethus Enslin, 1914

Nomenclature:

ATOMOSTETHUS Enslin, 1914

Eutomostethus ephippium (Panzer, 1798)

Nomenclature:

Tenthredo ephippium Panzer, 1798

dubius (Gmelin, 1790, Tenthredo) preocc.

inhabilis (Norton, 1861, Selandria)

Distribution: England, Scotland, Wales, Ireland

Eutomostethus gagathinus (Klug, 1816)

Nomenclature:

Tenthredo gagathina Klug, 1816

Distribution: England, Scotland, Wales

Eutomostethus luteiventris (Klug, 1816)

Nomenclature:

Tenthredo luteiventris Klug, 1816

fuscipennis (Serville, 1823, Tenthredo)

Distribution: England, Scotland, Wales, Ireland 
Eutomostethus nigrans (Konow, 1887)

Nomenclature:

Tomostethus nigrans Konow, 1887

nigrans Blank \& Taeger, 1998 preocc.

cinereipes (Klug, 1816): Cameron, 1882 misident.

ephippium (Panzer, 1798): misident.

Distribution: England, Scotland, Wales, Ireland

Notes: Added by Liston and O'Connor (2005).

\section{Eutomostethus punctatus (Konow, 1887)}

Nomenclature:

Tomostethus punctatus Konow, 1887

brachycera (Cameron, 1893, Tomostethus)

micans (Klug, 1816): Cameron, 1877 misident.

Distribution: England, Scotland, Wales

Genus Monophadnus Hartig, 1837

Monophadnus pallescens (Gmelin, 1790)

Nomenclature:

Tenthredo pallescens Gmelin, 1790

albipes (Gmelin, 1790, Tenthredo) preocc.

furvus Benson, 1930

Distribution: England, Scotland, Wales, Ireland

Genus Paracharactus MacGillivray, 1908

Nomenclature:

DICROSTEMA Benson, 1952

Paracharactus gracilicornis (Zaddach, 1859)

Nomenclature:

Selandria gracilicornis Zaddach, 1859

Distribution: England, Wales 
Genus Phymatocera Dahlbom, 1835

Nomenclature:

PECTINIA Brullé, 1846

HYPARGYRICUS MacGillivray, 1908

PHYTOMATOCERA: misspelling

Phymatocera aterrima (Klug, 1816)

Nomenclature:

Tenthredro aterrima Klug, 1816

robinsoni (Curtis, 1850, Selandria)

fuliginosa (Schrank, 1781): misident.

Distribution: England, Scotland, Wales

Genus Rhadinoceraea Konow, 1886

Rhadinoceraea micans (Schrank, 1781)

Nomenclature:

Tenthredo micans Klug, 1816

Distribution: England, Wales

Genus Stethomostus Benson, 1939

Stethomostus fuliginosus (Schrank, 1781)

Nomenclature:

Tenthredo fuliginosa Schrank, 1781

fuscus (Serville, 1823, Tenthredo)

Distribution: England, Scotland, Wales

Stethomostus funereus (Klug, 1816)

Nomenclature:

Tenthredo funerea Klug, 1816

Distribution: England 
Genus Tomostethus Konow, 1886

Tomostethus nigritus (Fabricius, 1804)

Nomenclature:

Tenthredo nigrita Fabricius, 1804

Distribution: England, Wales

Tribe Waldheimiini Smith, 1969

Genus Halidamia Benson, 1939

Halidamia affinis (Fallén, 1807)

Nomenclature:

Hylotoma affinis Fallén, 1907

hyalina (Klug, 1816, Tenthredo)

assimilis (Thomson, 1870, Blennocampa)

Distribution: England, Scotland, Wales, Ireland

Subfamily Nematinae Thomson, 1871

Notes: Unplaced species of Nematinae:

Nematus placidus Cameron, 1878

Placed by Konow (1905) as a synonym of Pristiphora leucopodia (Hartig, 1837), a species which has never been found in the British Isles. Konow probably did not examine the type of $N$. placidus, which according to Benson (1943b), is lost.

Tribe Cladiini Ashmead, 1898

Genus Cladius Illiger, 1807

Nomenclature:

PRIOPHORUS Dahlbom, 1835

TRICHIOCAMPUS Hartig, 1837

Cladius brullei (Dahlbom, 1835)

Nomenclature:

Priophorus brullei Dahlbom, 1835 
immunis Stephens, 1835

melanostigma (Stephens, 1835, Nematus)

tener Zaddach, 1859

tristis Zaddach, 1859

morio: misident.

Distribution: England, Scotland, Wales, Ireland

\section{Cladius compressicornis (Fabricius, 1804)}

Nomenclature:

Tenthredo compressicornis Fabricius, 1804

albipes (Fallén, 1808, Tenthredo) preocc.

pallipes Serville, 1823

padi: misident.

Distribution: England, Scotland, Wales, Ireland

Notes: The change in nomenclature is explained in Liston (2007).

\section{Cladius grandis (Serville, 1823)}

Nomenclature:

Nematus grandis Serville, 1823

viminalis (Fallén, 1808, Tenthredo) preocc.

Iuteicornis Stephens, 1835

Distribution: England, Scotland, Ireland

\section{Cladius pectinicornis (Geoffroy, 1785)}

Nomenclature:

Tenthredo pectinicornis Geoffroy, 1785

?difformis (Panzer, 1799, Tenthredo)

crassicornis (Stephens, 1835, Nematus)

Distribution: England, Scotland, Wales, Ireland

\section{Cladius pilicornis Curtis, 1833}

Distribution: England, Scotland, Wales, Ireland 
Cladius rufipes Serville, 1823

Nomenclature:

ulmi (Linnaeus, 1758): misident.

Distribution: England, Scotland, Ireland

Cladius ulmi (Linnaeus, 1758)

Nomenclature:

Tenthredo ulmi Linnaeus, 1759

eradiatus Hartig, 1837

laevifrons (Benson, 1936, Priophorus)

Distribution: England, Scotland, Wales, Ireland

Tribe Dineurini Ashmead, 1898

Genus Anoplonyx Marlatt, 1896

Anoplonyx destructor Benson, 1952

Nomenclature:

duplex (Lepeletier, 1823): misident.

Distribution: England, Scotland, Wales, Ireland

Genus Dineura Dahlbom, 1835

Dineura stilata (Klug, 1816)

Nomenclature:

Tenthredo stilata Klug, 1816

v-flavum (Cameron, 1882, Nematus)

Distribution: England, Scotland, Wales, Ireland

Dineura testaceipes (Klug, 1816)

Nomenclature:

Tenthredo testaceipes Klug, 1816

Distribution: England, Scotland, Wales, Ireland 
Dineura virididorsata (Retzius, 1783)

Nomenclature:

Tenthredo virididorsata Retzius, 1783

Distribution: England, Scotland, Wales, Ireland

Genus Hemichroa Stephens, 1835

Nomenclature:

LEPTOCERA Hartig, 1837

LEPTOCERCUS Thomson, 1871

Hemichroa australis (Serville, 1823)

Nomenclature:

Tenthredo australis Serville, 1823

alni (Linnaeus, 1767, Tenthredo) preocc.

luctuosa (Hill, 1773, Tenthredo) nom. ob.

Distribution: England, Scotland, Wales, Ireland

Hemichroa crocea (Geoffroy, 1785)

Nomenclature:

Tenthredo crocea Geoffroy, 1785

rufa (Panzer, 1799, Tenthredo) preocc.

stigma Stephens, 1835

Distribution: England, Scotland, Wales, Ireland

Genus Nematinus Rohwer, 1911

Notes: Unplaced species of Nematinus:

[antennatus (Cameron, 1877, Nematus)]

Nematinus acuminatus (Thomson, 1871)

Nomenclature:

Nematus acuminatus Thomson, 1871

Distribution: England, Scotland, Wales, Ireland 
Nematinus caledonicus (Cameron, 1882)

Nomenclature:

Nematus caledonicus Cameron, 1882

nigrosternatus Malaise, 1931

Distribution: England, Scotland, Ireland

Nematinus fuscipennis (Serville, 1823)

Nomenclature:

Nematus fuscipennis Serville, 1823

abdominalis (Fabricius, 1798): misident.

Distribution: England, Scotland, Wales, Ireland

Nematinus luteus (Panzer, 1803)

Nomenclature:

Nematus luteus Panzer, 1803

willigkiae R. Stein, 1926

willigkiae ssp. pilosus Benson, 1958

Distribution: England, Scotland, Wales, Ireland

\section{Nematinus steini Blank, 1998}

Nomenclature:

alneti (Bechstein \& Scharfenberg, 1805, Tenthredo) preocc.

bilineatus (Klug, 1819): Cameron, 1877, misident.

ruficapillus (Gmelin, 1790): Kirby, 1882, misident.

luteus (Panzer, 1803): misident.

Distribution: England, Scotland, Wales, Ireland

Genus Platycampus Schiødte, 1839

Nomenclature:

LEPTOPUS Hartig, 1837 preocc.

CAMPONISCUS Newman, 1869 
Platycampus luridiventris (Fallén, 1808)

Nomenclature:

Tenthredo luridiventris Fallén, 1808

niger (Stephens, 1835, Nematus)

alnivorus (Hartig, 1840, Nematus)

healaei (Newman, 1869, Camponiscus)

Distribution: England, Scotland, Wales, Ireland

Tribe Hoplocampini Konow, 1890

Genus Hoplocampa Hartig, 1837

Hoplocampa alpina (Zetterstedt, 1838)

Nomenclature:

Tenthredo alpina Zetterstedt, 1838

pallida (Newman, 1837, Selandria) preocc.

Distribution: England, Scotland, Ireland

Hoplocampa ariae Benson, 1933

Distribution: England, Ireland

Hoplocampa brevis (Klug, 1816

Nomenclature:

Tenthredo brevis Klug, 1816

Distribution: England

Hoplocampa chrysorrhoea (Klug, 1816)

Nomenclature:

Tenthredo chrysorrhoea Klug, 1816

Distribution: England, Scotland, Wales, Ireland

Hoplocampa crataegi (Klug, 1816)

Nomenclature:

Tenthredo crataegi Klug, 1816 
plagiata (Klug, 1816): Cameron, 1885 misident.

Distribution: England, Scotland, Wales, Ireland

Hoplocampa flava (Linnaeus, 1761)

Nomenclature:

Tenthredo flava Linnaeus, 1760

ferruginea (Fabricius, 1804): misident.

minuta (Christ, 1791): misident.

Distribution: England, Scotland, Wales, Ireland

Hoplocampa fulvicornis (Panzer, 1801)

Nomenclature:

Tenthredo fulvicornis Panzer, 1801

rutilicornis (Klug, 1816, Tenthredo)

Distribution: England, Scotland, Wales, Ireland

Hoplocampa pectoralis Thomson, 1871

Nomenclature:

gallicola Cameron, 1877

Distribution: England, Scotland, Wales, Ireland

Hoplocampa testudinea (Klug, 1816)

Nomenclature:

Tenthredo testudinea Klug, 1816

Distribution: England, Scotland, Wales

Tribe Mesoneurini Zombori, 1982

Genus Mesoneura Hartig, 1837

Mesoneura opaca (Fabricius, 1775)

Nomenclature:

Tenthredo opaca Fabricius, 1775

verna (Klug, 1816, Tenthredo) 
biloba (Stephens, 1835, Selandria)

selandriiformis (Cameron, 1875, Dineura)

Distribution: England, Scotland, Wales, Ireland

Tribe Nematini Thomson, 1871

Genus Amauronematus Konow, 1890

Nomenclature:

PONTOPRISTIA Malaise, 1921

BRACHYCOLUMA Strand, 1929

BRACHYCOLUS Konow, 1895 preocc.

DECANEMATUS Malaise, 1931

Amauronematus abnormis (Holmgren, 1883)

Nomenclature:

Nematus abnormis Holmgren, 1883

tolli Konow, 1907

aulatus MacGillivray, 1919

Distribution: Scotland

Amauronematus amentorum (Förster, 1854)

Nomenclature:

Nematus amentorum Förster, 1854

suavis (Ruthe, 1859, Nematus)

kamtchaticus (Malaise, 1931, Pontopristia)

Distribution: England, Scotland

Amauronematus amplus Konow, 1895

Distribution: England, Scotland, Ireland

Amauronematus fasciatus Konow, 1897

Nomenclature:

perkinsi Benson, 1933

variabilis Malaise, 1931: misident. 
Distribution: England, Scotland, Wales, Ireland

\section{Amauronematus godmani Benson, 1955}

Distribution: Scotland

Notes: Specimens identified by Benson as this species in NMS, from Switzerland (type locality) and Scotland, do not seem to be conspecific. They both belong to the Amauronematus variator (Ruthe) species group. The identity of Scottish specimens requires further study.

\section{Amauronematus hedstroemi Malaise, 1931}

Nomenclature:

rex Benson, 1948

tillbergi Malaise, 1920: misident.

Distribution: England, Scotland, Wales, Ireland

Notes: See Lindqvist (1972) on taxonomy of this species and A. tillbergi Malaise, 1920.

\section{Amauronematus histrio (Serville, 1823)}

\section{Nomenclature:}

Nematus histrio Serville, 1823

rufescens (Hartig 1837, Nematus)

glenelgensis (Cameron, 1882, Nematus)

Distribution: England, Scotland, Ireland

Notes: British and Irish specimens at present identified as this species or as $A$. stenogaster may include other taxa not yet recorded in the British Isles. See revision of North European taxa by Schmidt (1997).

\section{Amauronematus humeralis (Serville, 1823)}

\section{Nomenclature:}

Nematus humeralis Serville, 1823

terminalis Malaise, 1931

Distribution: England, Scotland, Wales 
Amauronematus krausi Taeger \& Blank, 1998

Nomenclature:

puniceus (Christ, 1791): misident.

Distribution: England

Amauronematus lateralis Konow, 1896

Nomenclature:

trautmanni Enslin, 1919

cameroni Perkins, 1929

piliserra Lindqvist, 1943

imperfectus (Zaddach, 1876): Cameron, 1885 misident.

Distribution: England, Scotland, Wales, Ireland

Amauronematus leucolenus (Brischke, 1883)

Nomenclature:

Nematus leucolenus Brischke, 1883

saarineni (Lindqvist, 1935): Benson, 1948 misident.

leucolaenus: Benson, 1958 misspelling

Distribution: England, Ireland

Amauronematus longiserra (Thomson, 1863)

Nomenclature:

Nematus longiserra Thomson, 1863

Distribution: England, Scotland, Wales

Amauronematus mcluckieae Benson, 1935

Nomenclature:

pustulatus Lindqvist, 1962

arcticola Enslin, 1915: misident.

Distribution: Scotland

Notes: See Liston et al. (2012) on the spelling of the species name. 
Amauronematus miltonotus (Zaddach, 1883)

Nomenclature:

Nematus miltonotus Zaddach, 1883

Distribution: England, Wales

Amauronematus mimus Schmidt, 1997

Distribution: Scotland

Notes: Added by Liston et al. (2012).

Amauronematus mundus Konow, 1895

Distribution: England, Scotland, Wales, Ireland

Amauronematus sagmarius Konow, 1895

Distribution: England, Scotland, Ireland

Amauronematus semilacteus (Zaddach, 1883)

Nomenclature:

Nematus semilacteus Zaddach, 1883

Distribution: Scotland

Amauronematus stenogaster (Förster, 1854)

Nomenclature:

Nematus stenogaster Förster, 1854

festivus Saarinen, 1950

fallax (Serville, 1823): misident.

Distribution: England, Scotland, Wales, Ireland

Amauronematus toeniatus (Serville, 1823)

Nomenclature:

Nematus toeniatus Serville, 1823

taeniatus (Lepeletier, 1823, Nematus) preocc.

aemulus Konow, 1895

zetterstedti Malaise, 1920 
alpicola Konow, 1895: misident.

Distribution: England, Scotland

Amauronematus tristis Lindqvist, 1959

Nomenclature:

sempersolis Kiaer, 1898: misident.

Notes: Added by Knight \& Liston (in prep.).

Amauronematus tunicatus (Zaddach, 1883)

Nomenclature:

Nematus tunicatus Zaddach, 1883

Distribution: England, Ireland

Amauronematus viduatus (Zetterstedt, 1838)

Nomenclature:

Tenthredo viduata Zetterstedt, 1838

notatus (Förster, 1854, Nematus)

longiserra (Thomson, 1863): Cameron, 1876 misident.

Distribution: England, Scotland, Wales, Ireland

Amauronematus vittatus (Serville, 1823)

Nomenclature:

Nematus vittatus Serville, 1823

crispus Benson, 1948

Distribution: England, Scotland, Wales, Ireland

\section{Genus Craesus Leach, 1817}

Nomenclature:

CROESUS Curtis, 1824: misspelling

\section{Craesus alniastri (Scharfenberg, 1805)}

Nomenclature:

Tenthredo alniastri Scharfenberg, 1805

varus (Villaret, 1832, Nematus) 
Distribution: England, Scotland, Wales, Ireland

Craesus brischkei (Zaddach, 1876)

Nomenclature:

Nematus brischkei Zaddach, 1876

Distribution: England

Craesus latipes (Villaret, 1832)

Nomenclature:

Nematus latipes Villaret, 1832

Distribution: England, Scotland, Wales, Ireland

\section{Craesus septentrionalis (Linnaeus, 1758)}

Nomenclature:

Tenthredo septentrionalis Linnaeus, 1758

stephensii Newman, 1837

Distribution: England, Scotland, Wales, Ireland

\section{Genus Euura Newman, 1837}

Nomenclature:

CRYPTOCAMPUS Hartig, 1837

GEMMURA Smith, 1968

\section{Euura amerinae (Linnaeus, 1758)}

Nomenclature:

Cynips amerinae Linnaeus, 1758

salicispentandrae (Retzius, 1783, Tenthredo)

medullarius (Hartig, 1837, Nematus)

saliceti (Fallén, 1808, Tenthredo)

mucronata (Hartig, 1837): Vollenhoven, 1871 misident.

Distribution: England, Scotland, Ireland 


\section{Euura atra (Jurine, 1807)}

Nomenclature:

Pteronus ater Jurine, 1807

Distribution: England, Scotland, Wales, Ireland

Notes: Regarded as a group of sibling (biological) species by Kopelke (1996), Kopelke (2000). These are very difficult, perhaps impossible, to distinguish using adult morphological characters. According to records of galls on various identified Salix species, at least the following three species belonging to the group occur in the British Isles: E. auritae, E. purpureae and E. weiffenbachii.

\section{Euura auritae Kopelke, 2000}

Distribution: Scotland

\section{Euura mucronata (Hartig, 1837)}

Nomenclature:

Nematus mucronatus Hartig, 1837

gallae Newman, 1837

?nigritarsis (Cameron, 1885, Cryptocampus)

saliceti (Fallén, 1808): misident.

Distribution: England, Scotland, Wales, Ireland

Notes: According to Kopelke (2001), several biological species are included under this name. A number of these seem to occur in the British Isles, but an alternative view on some of the taxa proposed by Kopelke has been offered by Nyman (2002).

\section{Euura purpureae Kopelke, 1996}

Distribution: Ireland

Notes: The only known record in the British Isles is based on the specimens mentioned by O'Connor et al. (1997) under the name Euura atra, as having been reared from Salix purpurea.

\section{Euura testaceipes (Brischke, 1883)}

\section{Nomenclature:}

Cryptocampus testaceipes Brischke, 1883

cynips Newman, 1837 nom. ob.

Distribution: England, Scotland 
Euura venusta (Brischke, 1883)

Nomenclature:

Cryptocampus venustus Brischke, 1883

Distribution: England, Scotland, Wales

Euura weiffenbachii Ermolenko, 1988

Distribution: Scotland, Ireland

Notes: Added by Liston and O'Connor (2005).

Genus Nematus Panzer, 1801

Subgenus Kontuniemiana Lacourt, 1998

Nematus (Kontuniemiana) leucotrochus Hartig, 1837

Nomenclature:

maculiventris Hartig, 1840

approximatus Förster, 1854

consobrinus Vollenhoven, 1871

cognatus (Lindqvist, 1957, Pteronidea)

Distribution: England, Scotland, Wales, Ireland

Nematus (Kontuniemiana) olfaciens Benson, 1953

Distribution: England, Scotland

Nematus (Kontuniemiana) ribesii (Scopoli, 1763)

Nomenclature:

Tenthredo ribesii Scopoli, 1763

dimidiatus Serville, 1823

trimaculatus Serville, 1823

grossulariae Moore, 1831

ventricosus (Bouché, 1834, Tenthredo)

macrocerus Hartig, 1840

ribis Dufour, 1847

Distribution: England, Scotland, Wales, Ireland 
Subgenus Nematus Panzer, 1801

Nomenclature:

HYPOLAEPUS W. F. Kirby, 1882

HOLCOCNEME Konow, 1890

Nematus (Nematus) caeruleocarpus Hartig, 1837

Nomenclature:

brevispinis Förster, 1854

brachyacanthus Thomson, 1863

sulcipes Hartig, 1837

coeruleocarpus: misspelling

Distribution: England, Scotland, Ireland

Nematus (Nematus) lucidus (Panzer, 1801)

Nomenclature:

Tenthredo lucida Panzer, 1801

Distribution: England, Scotland, Wales, Ireland

Nematus (Nematus) vicinus Serville, 1823

Nomenclature:

longispinus Kriechbaumer, 1885

crassus (Fallén, 1808, Tenthredo) preocc.

sulcipes (Hartig, 1837): misident.

Distribution: England, Scotland, Wales, Ireland

Subgenus Pteronidea Rohwer, 1911

Nomenclature:

LINDQVISTIA Lacourt, 1998

Nematus (Pteronidea) bergmanni Dahlbom, 1835

Nomenclature:

pallicarpus Hartig, 1837

curtispina Thomson, 1871

pallens (Enslin, 1916, Lygaeonematus) 
vernalis (Lindqvist, 1937, Pteronidea)

Distribution: England, Scotland, Wales, Ireland

Nematus (Pteronidea) bipartitus Serville, 1823

Distribution: England, Scotland, Wales

Nematus (Pteronidea) brevivalvis Thomson, 1871

Nomenclature:

kriegeri (Konow, 1903, Pteronus)

spurcus (Konow, 1904, Amauronematus)

absimilis (Lindqvist, 1939, Pteronidea)

Distribution: England, Scotland, Wales, Ireland

Nematus (Pteronidea) cadderensis Cameron, 1875

Nomenclature:

macroserratus (Lindqvist, 1943, Pteronidea)

Distribution: England, Scotland, Ireland

Nematus (Pteronidea) dorsatus Cameron, 1875

Distribution: Scotland, Wales

Nematus (Pteronidea) fagi Zaddach, 1876

Distribution: England, Scotland, Wales

Nematus (Pteronidea) fahraei Thomson, 1862

Distribution: England, Scotland

Nematus (Pteronidea) ferrugineus Förster, 1854

Nomenclature:

glottianus Cameron, 1882

Distribution: England, Scotland, Ireland 
Nematus (Pteronidea) flavescens Stephens, 1835

Distribution: England, Scotland, Wales, Ireland

Nematus (Pteronidea) frenalis Thomson, 1888

Nomenclature:

fastosus (Konow, 1904, Pteronus)

fastuosus: misspelling

Distribution: Scotland, Ireland

Nematus (Pteronidea) fuscomaculatus Förster, 1854

Nomenclature:

strongylogaster Cameron, 1878

dossuarius (Konow, 1904, Pteronus)

Distribution: England, Scotland

Nematus (Pteronidea) hypoxanthus Förster, 1854

Nomenclature:

orbitalis Cameron, 1884

nigronotus (Lindqvist, 1957, Pteronidea)

Distribution: England, Scotland, Wales, Ireland

Nematus (Pteronidea) incompletus Förster, 1854

Nomenclature:

pulchellus Cameron, 1882

segmentarius Förster, 1854: misident.

Distribution: England, Scotland, Ireland

Nematus (Pteronidea) jugicola Thomson, 1871

Nomenclature:

karvoneni (Lindqvist, 1969, Pteronidea)

Distribution: England, Scotland 
Nematus (Pteronidea) leionotus (Benson, 1933)

Nomenclature:

Pteronidea leionota Benson, 1933

Distribution: England, Scotland, Ireland

Nematus (Pteronidea) melanocephalus Hartig, 1837

Distribution: England, Scotland, Wales, Ireland

Nematus (Pteronidea) miliaris (Panzer, 1797)

Nomenclature:

Tenthredo miliaris Panzer, 1797

capreae (Linnaeus, 1758): misident.

croceus (Fallén, 1808): misident.

testaceus Stephens, 1835

Distribution: England, Scotland, Wales, Ireland

Nematus (Pteronidea) monticola Thomson, 1871

Nomenclature:

perkioemaekii (Lindqvist, 1960, Pachynematus)

?pschornwalcheri Muche, 1972

similator Förster, 1854: misident.

Distribution: England, Scotland

Nematus (Pteronidea) myosotidis (Fabricius, 1804)

Nomenclature:

Tenthredo myosotidis Fabricius, 1804

ambiguus Förster, 1854

papillosus (Retzius, 1783): misident.

Distribution: England, Scotland, Wales, Ireland

Nematus (Pteronidea) nigricornis Serville, 1823

Distribution: England, Scotland, Wales, Ireland 
Nematus (Pteronidea) nubium (Benson, 1935)

Nomenclature:

Pteronidea nubium Benson, 1935

roberti (Lindqvist, 1958, Pteronidea)

Distribution: Scotland

Nematus (Pteronidea) oligospilus Förster, 1854

Nomenclature:

salicivorus Cameron, 1882

miliaris (Panzer, 1797): Cameron, 1885 misident.

capreae (Linnaeus, 1758): misident.

Distribution: England, Scotland, Wales, Ireland

Nematus (Pteronidea) papillosus (Retzius, 1783)

Nomenclature:

Tenthredo papillosa Retzius, 1783

melanaspis Hartig, 1840

?maculiger Cameron, 1882

sveae (Lindqvist, 1958, Pteronidea)

Distribution: England, Scotland, Wales, Ireland

Nematus (Pteronidea) pavidus Serville, 1823

Nomenclature:

wttewaalli Vollenhoven, 1862

cameronii Dalla Torre, 1894

aurantiacus Hartig, 1837: misident.

similator Förster, 1854: misident.

Distribution: England, Scotland, Wales, Ireland

Nematus (Pteronidea) poecilonotus Zaddach, 1876

Nomenclature:

viridescens Cameron, 1885

subnitens (Lindqvist, 1957, Pteronidea) 
palliatus Thomson, 1863: misident.

Distribution: England, Scotland, Wales, Ireland

Nematus (Pteronidea) pravus (Konow, 1895)

Nomenclature:

Amauronematus pravus Konow, 1895

Distribution: Scotland

Notes: Added by Knight and Liston (in prep.). Some differences between Scottish individuals and the Estonian lectotype require further investigation.

Nematus (Pteronidea) pseudodispar (Lindqvist, 1969)

Nomenclature:

Pteronidea pseudodispar Lindqvist, 1969

Distribution: England, Scotland

Notes: Added by Knight and Liston (in prep.).

Nematus (Pteronidea) reticulatus Holmgren, 1883

Nomenclature:

arcticus Thomson, 1871 preocc.

arcticola Dalla Torre, 1894

forsiusi (Enslin, 1915, Pontania)

alsius (Benson, 1935, Amauronematus)

Distribution: Scotland

Nematus (Pteronidea) salicis (Linnaeus, 1758)

Nomenclature:

Tenthredo salicis Linnaeus, 1758

Distribution: England, Scotland, Wales

Nematus (Pteronidea) spiraeae Zaddach, 1883

Distribution: England, Scotland, Wales, Ireland 
Nematus (Pteronidea) stichi (Enslin, 1913)

Nomenclature:

Pteronidea stichi Enslin, 1913

testaceus Thomson, 1871 preocc.

fuscarima (Benson, 1933, Pteronidea)

pallescens Hartig, 1837: Cameron, 1877 misident.

Distribution: England, Scotland, Ireland

Nematus (Pteronidea) sylvestris Cameron, 1884

Nomenclature:

punctiscuta Hellén, 1948

ponojensis Hellén, 1948

silvestris: misspelling

Distribution: England, Scotland

Nematus (Pteronidea) tibialis Newman, 1837

Nomenclature:

hortensis Hartig, 1837

trilineatus Norton, 1867

similaris Norton, 1880

robiniae Forbes, 1885

Distribution: England

Nematus (Pteronidea) umbratus Thomson, 1871

Nomenclature:

collinus Cameron, 1882

similis (Forsius, 1911, Pteronidea)

verrucosae (Kontuniemi, 1966, Pteronidea)

Distribution: England, Scotland, Ireland

Nematus (Pteronidea) viridis Stephens, 1835

Nomenclature:

prasinus Hartig, 1837 
brevivalvis Thomson, 1871: Konow, 1890 misident.

dispar Zaddach, 1876: misident.

bergmanni Dahlbom, 1835: misident.

Distribution: England, Scotland, Wales, Ireland

Nematus (Pteronidea) viridissimus Möller, 1882

Nomenclature:

?glutinosae Cameron, 1882

polyspilus Förster, 1854: misident.

Distribution: England, Scotland, Wales, Ireland

Notes: According to Lindqvist (1962), N. glutinosae (description probably predates that of $N$. viridissimus by a few months) is not conspecific with $N$. viridissimus.

\section{Genus Pachynematus Konow, 1890}

Nomenclature:

PIKONEMA Ross, 1937

LARINEMATUS Zhelochovtsev, 1988

POLYNEMATUS Zhelochovtsev, 1988

EPICENEMATUS Lacourt, 1998

\section{Pachynematus albipennis (Hartig, 1837)}

Nomenclature:

Nematus albipennis Hartig, 1837

sannio (Konow, 1903, Pachynematus)

Distribution: England, Scotland, Wales, Ireland

\section{Pachynematus annulatus (Gimmerthal, 1834)}

Nomenclature:

Nematus annulatus Gimmerthal, 1834

flavipennis (Cameron, 1876, Nematus)

arcticus (Thomson, 1871): Cameron, 1878 misident.

rumicis (Linnaeus, 1758): Fallén, 1808 misident.

rumicis: Benson, 1958 
Distribution: England, Scotland, Ireland

Pachynematus calcicola Benson, 1948

Nomenclature:

chambersi Benson, 1948

laevigatus Zaddach, 1883: Benson, 1967 misident.

Distribution: England, Ireland

\section{Pachynematus clibrichellus (Cameron, 1882)}

Nomenclature:

Nematus clibrichellus Cameron, 1878

thomsoni (Cameron, 1882, Nematus)

clibrichensis (Cameron, 1885, Nematus): unjustified emendation

hyperboreus (Thomson, 1871): Cameron, 1878 misident.

Distribution: England, Scotland, Wales

\section{Pachynematus clitellatus (Serville, 1823)}

Nomenclature:

Nematus clitellatus Serville, 1823

trisignatus (Förster, 1854, Nematus)

foveolatus Konow, 1903

truncatus Benson, 1948

extensicornis (Norton, 1861): misident.

Distribution: England, Scotland, Wales, Ireland

Notes: Taeger and Blank (1998) regard P. clitellatus and $P$. fallax as morphologically highly variable species, which together include most of the previously described $\mathrm{W}$. Palaearctic taxa. Their arguments for this are well reasoned, but at present it seems prudent to continue to regard several of the more distinctive morphological segregates, treated as species by Benson (1958), as separate taxa, because morphologically intermediate specimens are rare (at least in the male sex). The taxonomy of the whole group requires further intensive work, including genetic analysis. According to Taeger and Blank (1998) P. kirbyi, treated here as valid, is a synonym of $P$. clitellatus. 
Pachynematus fallax (Serville, 1823)

Nomenclature:

Nematus fallax Serville, 1823

xanthocarpus (Hartig, 1840, Nematus)

virginalis Liston, 1982

Distribution: England, Scotland, Ireland

Notes: See above, under P. clitellatus. According Taeger and Blank (1998), P. sulcatus and $P$. calcicola are synonyms of $P$. xanthocarpus (= P. fallax).

\section{Pachynematus glabriceps Lindqvist, 1949}

Nomenclature:

parvilabris (Thomson, 1863): misident.

Distribution: England

Pachynematus imperfectus (Zaddach, 1876)

Nomenclature:

Nematus imperfectus Zaddach, 1876

Distribution: England, Scotland, Wales

Pachynematus kirbyi (Dahlbom, 1835)

Nomenclature:

Nematus kirbyi Dahlbom, 1835

flaviventris (Hartig, 1840, Nematus)

melanocerus (Hartig, 1840, Nematus)

diaphanus (Eversmann, 1847, Nematus)

umbripennis (Eversmann, 1847, Nematus)

turgidus (Zaddach, 1876, Nematus)

Distribution: England, Scotland, Wales, Ireland

\section{Pachynematus lichtwardti Konow, 1903}

Nomenclature:

apicalis (Hartig, 1837, Nematus) preocc.

Distribution: England, Scotland, Wales, Ireland 


\section{Pachynematus moerens (Förster 1854)}

Nomenclature:

Nematus moerens Förster, 1854

pygostolus (Förster, 1854, Nematus)

pleuralis (Thomson, 1863, Nematus)

pullus Konow, 1903

torridonensis Liston, 1980

Distribution: England, Scotland, Wales, Ireland

Pachynematus montanus (Zaddach, 1883)

Nomenclature:

Nematus montanus Zaddach, 1883

Distribution: England, Scotland, Wales, Ireland

Pachynematus obductus (Hartig, 1837)

Nomenclature:

Nematus obductus Hartig, 1837

conductus (Ruthe, 1859, Nematus)

graminis (Cameron, 1874, Nematus)

Distribution: England, Scotland, Wales, Ireland

\section{Pachynematus scutellatus (Hartig, 1837)}

Nomenclature:

Nematus scutellatus Hartig, 1837

Pikonema scutellatum: Lacourt, 1999

Distribution: England, Scotland, Wales, Ireland

\section{Pachynematus smithae Ross, 1945}

Nomenclature:

angustatus Lindqvist, 1949

smithiae: misspelling

Distribution: England, Scotland 
Pachynematus sulcatus Benson, 1948

Distribution: England, Scotland

Pachynematus vagus (Fabricius, 1781)

Nomenclature:

Tenthredo vaga Fabricius, 1781

leucogaster (Hartig, 1840, Nematus)

inconspicuus (W. F. Kirby, 1882, Nematus)

Distribution: England, Scotland, Wales, Ireland

Genus Phyllocolpa Benson, 1960

Phyllocolpa acutiserra (Lindqvist, 1949)

Nomenclature:

Pontania acutiserra Lindqvist, 1949

Distribution: Scotland

Phyllocolpa alienata (Förster, 1854)

Nomenclature:

Nematus alienatus Förster, 1854

coriacea (Benson, 1953): misident.

Distribution: Scotland

Phyllocolpa anglica (Cameron, 1877

Nomenclature:

Nematus anglicus Cameron, 1877

nigrolineata (Cameron, 1879, Nematus)

Distribution: England, Ireland

Notes: Three morphologically well distinguished Phyllocolpa species feed on Salix viminalis in Europe (Vikberg 2010a). Benson (1958) had already distinguished these under the names P. piliserra, P. scotaspis and P. anglica. Kopelke (2007b) stated that only two European species occur on this host, and synonymised Nematus anglicus and $N$. nigrolineatus with $P$. scotaspis, thus leaving the taxon formerly referred to as $P$. anglica without a valid name. The opinions of Benson and Vikberg are followed here. 
Phyllocolpa carinifrons (Benson, 1940)

Nomenclature:

Pontania carinifrons Benson, 1940

excavata (Marlatt, 1896): misident.

destricta (MacGillivray, 1923): misident.

apicifrons (Malaise, 1931): misident.

Distribution: England, Scotland, Ireland

Notes: Taxonomy follows Kopelke (2007a).

\section{Phyllocolpa erythropyga (Förster, 1854)}

Nomenclature:

Nematus erythropygus Förster, 1854

leucosticta (Hartig, 1837): misident.

Distribution: Scotland

Notes: Added by Liston et al. (2012).

\section{Phyllocolpa ischnocera (Thomson, 1863)}

Nomenclature:

Nematus ischnocerus Thomson, 1863

leucostigma (Cameron, 1876, Nematus)

Distribution: Scotland

Notes: Taxonomy follows Kopelke (2007a).

\section{Phyllocolpa leucapsis (Tischbein, 1846)}

\section{Nomenclature:}

Nematus leucapsis Tischbein, 1846

coriacea (Benson, 1953, Nematus)

Distribution: England, Scotland, Wales, Ireland

Notes: According to Kopelke (2007c) P. coriacea is a synonym of $P$. leucapsis (as stated in the abstract and table 1, whereas the synonymy with $P$. alienata on p. 153 is wrong; J.-P. Kopelke, pers. comm.). The concept of $P$. leucapsis in Kopelke (2007c) is different to that of Benson (1958) and most other previous taxonomists. 
Phyllocolpa leucosticta (Hartig, 1837)

Nomenclature:

Nematus leucostictus Hartig, 1837

sharpi (Cameron, 1876, Nematus)

Distribution: England, Scotland, Wales, Ireland

Notes: According to Kopelke (2007a), P. erythropyga (Förster, 1854) has previously been confused with $P$. leucosticta. Whilst $P$. erythropyga was recorded in the British Isles by Liston et al. (2012), records of $P$. leucosticta require validation.

\section{Phyllocolpa oblita (Serville, 1823)}

Nomenclature:

Nematus oblitus Serville, 1823

puella (Thomson, 1871, Nematus)

Distribution: England, Wales

\section{Phyllocolpa piliserra (Thomson, 1863)}

Nomenclature:

Nematus piliserra Thomson, 1863

xanthogaster (Förster, 1854): Cameron, 1877 misident.

Distribution: England, Scotland, Ireland

\section{Phyllocolpa plicalapponum Kopelke, 2007}

Distribution: Scotland

Notes: Added by Liston et al. (2012).

\section{Phyllocolpa plicaphylicifolia Kopelke, 2007}

Distribution: Scotland

Notes: Added by Liston et al. (2012).

Phyllocolpa polita (Zaddach, 1883)

Nomenclature:

Nematus politus Zaddach, 1883

Distribution: Scotland 
Notes: Regarded as a valid species by Kopelke (2003), Kopelke (2007a). Material from

Scotland was mentioned by Brischke (1884).

\section{Phyllocolpa prussica (Zaddach, 1883)}

Nomenclature:

Nematus prussicus Zaddach, 1883

Distribution: Scotland

Notes: Added by Liston et al. (2012).

\section{Phyllocolpa scotaspis (Förster, 1854)}

Nomenclature:

Nematus scotaspis Förster, 1854

westermanni (Boheman, 1852, Nematus) nom. ob.

westermanni (Thomson, 1863, Nematus)

Distribution: England, Scotland, Ireland

\section{Genus Pontania Costa, 1852}

Nomenclature:

EUPONTANIA Zinovjev, 1985

TUBPONTANIA Vikberg, 2010

Notes: Subsequent to the revision of the viminalis species group by Kopelke (1991), numerous important taxonomic and nomenclatural changes, followed here, were proposed by Vikberg (2003) and Vikberg and Zinovjev (2006).

\section{Pontania anomaloptera (Förster, 1854)}

Nomenclature:

Nematus anomalopterus Förster, 1854

tuberculata (Benson, 1953, Nematus)

Distribution: England, Scotland, Wales, Ireland

Notes: Taxonomy of the species follows Vikberg (2010b) (under Tubpontania anomaloptera). 
Pontania aquilonis Benson, 1941

Nomenclature:

algida Benson, 1941

Distribution: Scotland

Pontania arbusculae Benson, 1941

Distribution: Scotland

Pontania arcticornis Konow, 1904

Nomenclature:

phylicifoliae Forsius, 1920

hepatimaculae Malaise, 1920

Distribution: England, Scotland, Ireland

Pontania brevicornis (Förster, 1854)

Nomenclature:

Nematus brevicornis Förster, 1854

curticornis (Cameron, 1885, Nematus)

saliciscinereae (Retzius, 1783): misident.

kriechbaumeri Konow, 1901: misident.

pedunculi (Hartig, 1837): Kopelke 1991 misident.

Distribution: England, Scotland

Pontania bridgmanii (Cameron, 1883)

Nomenclature:

Nematus bridgmanii Cameron, 1883

capreae (Linnaeus, 1758): misident.

Distribution: England, Scotland, Wales, Ireland

Pontania collactanea (Förster, 1854)

Nomenclature:

Nematus collactaneus Förster, 1854

vacciniellus (Cameron, 1876, Nematus) 
Distribution: England, Scotland, Ireland

Pontania crassipes (Thomson, 1871)

Nomenclature:

Nematus crassipes Thomson, 1871

lapponica Malaise, 1920

Distribution: Scotland

Pontania dolichura (Thomson, 1871)

Nomenclature:

Nematus dolichurus Thomson, 1871

lapponicola Kopelke, 1994

Distribution: Scotland

Notes: Added on the basis of records of galls of $P$. dolichura from Salix lapponum in

Benson (1954). See Vikberg and Malinen (2012) on taxonomy.

Pontania femoralis (Cameron, 1876)

Nomenclature:

Nematus femoralis Cameron, 1876

robbinsi Benson, 1935

dolichura (Thomson, 1871): misident.

Distribution: England, Scotland

Notes: See Vikberg and Malinen (2012) on taxonomy.

\section{Pontania herbaceae (Cameron, 1876)}

Nomenclature:

Nematus herbaceae Cameron, 1876

crassipes (Thomson, 1871): misident.

Distribution: England, Scotland, Wales, Ireland

Pontania myrsiniticola Kopelke, 1991

Distribution: Scotland

Notes: Added by Liston and Blank (2006). 
Pontania nigricantis Kopelke, 1986

Nomenclature:

dolichura (Thomson, 1871): misident.

Distribution: England, Scotland

Notes: Added on the basis of records of galls of $P$. dolichura from Salix nigricans in Benson (1954).

Pontania pedunculi (Hartig, 1837)

Nomenclature:

Nematus pedunculi Hartig, 1837

bella (Zaddach, 1876, Nematus)

baccarum (Cameron, 1876, Nematus)

pusilla Lindqvist, 1964

gallarum (Hartig, 1837): Kopelke, 1991 misident.

Distribution: England, Scotland, Ireland

Pontania proxima (Serville, 1823)

Nomenclature:

Nematus proximus Serville, 1823

gallicola (Stephens, 1835, Nematus)

flavipes (Cameron, 1885, Euura)

capreae (Linnaeus, 1758): misident.

Distribution: England, Scotland, Wales, Ireland

Pontania purpureae (Cameron, 1884)

Nomenclature:

Nematus purpureae Cameron, 1884

Distribution: England

Pontania pustulator Forsius, 1923

Distribution: England, Scotland 
Pontania saliciscinereae (Retzius, 1783)

Nomenclature:

Tenthredo saliciscinereae Retzius, 1783

gallarum (Hartig, 1837, Nematus)

aestiva (Thomson, 1862, Nematus)

harrisoni Benson, 1940

varia Kopelke, 1991

Distribution: England, Scotland

Notes: Nomenclature follows Blank et al. (2009).

Pontania samolad Malaise, 1920

Distribution: Scotland

Pontania triandrae Benson, 1941

Nomenclature:

capreae (Linnaeus, 1758): misident.

Distribution: England, Scotland, Ireland

Pontania vesicator (Bremi-Wolf, 1849)

Nomenclature:

Nematus vesicator Bremi-Wolf, 1849

helicina (Brischke, 1850, Nematus)

Distribution: England, Scotland, Ireland

Pontania viminalis (Linnaeus, 1758)

Nomenclature:

Cynips viminalis Linnaeus, 1758

vollenhoveni (Cameron, 1874, Nematus)

?interstitialis (Cameron, 1876, Nematus)

hungarica Enslin, 1918

Distribution: England, Scotland, Wales, Ireland 
Tribe Pristiphorini Vikberg, 1982

Genus Pristicampus Zinovjev, 1993

Pristicampus arcticus (Lindqvist, 1958)

Nomenclature:

Mesoneura arctica Lindqvist, 1959

Pachynematus arcticus: Benson, 1961

Distribution: Scotland

Genus Pristiphora Latreille, 1810

Notes: Species of Pristiphora removed from the British and Irish list:

[karvoneni (Lindqvist, 1952, Lygaeonematus)] Recorded by Liston (1983a) but based on a misidentification.

[micronematica Malaise, 1931] Recorded by Liston (1982) but based on a misidentification.

Subgenus Lygaeonematus Konow, 1890

Pristiphora (Lygaeonematus) abietina (Christ, 1791)

Nomenclature:

Tenthredo abietina Christ, 1791

pini (Retzius, 1783, Tenthredo) preocc.

Distribution: England, Scotland, Wales, Ireland

Pristiphora (Lygaeonematus) compressa (Hartig, 1837)

Nomenclature:

Nematus compressus Hartig, 1837

Distribution: England, Scotland

Pristiphora (Lygaeonematus) decipiens (Enslin, 1916)

Nomenclature:

Lygaeonematus decipiens Enslin, 1916

Distribution: England, Scotland 
Notes: Added Liston (1981b).

Pristiphora (Lygaeonematus) erichsonii (Hartig, 1837)

Nomenclature:

Nematus erichsonii Hartig, 1837

leachii (Dahlbom, 1835, Nematus): suppressed

Distribution: England, Scotland, Wales, Ireland

Pristiphora (Lygaeonematus) glauca Benson, 1954

Nomenclature:

?laricivora (Takagi, 1931, Pachynematus) preocc.

Distribution: England, Wales

Pristiphora (Lygaeonematus) pseudodecipiens Beneš \& Kristek, 1976

Nomenclature:

decipiens (Enslin, 1916): misident.

Distribution: England, Scotland

Notes: Added by Beneš and Krístek (1976).

Pristiphora (Lygaeonematus) saxesenii (Hartig, 1837)

Nomenclature:

Nematus saxesenii Hartig, 1837

thalenhorsti Wong, 1975

Distribution: England, Scotland, Wales

Pristiphora (Lygaeonematus) subarctica (Forsslund, 1936)

Nomenclature:

Lygaeonematus subarcticus Forsslund 1936

pseudosaxesenii Lindqvist, 1968

Distribution: England 
Pristiphora (Lygaeonematus) wesmaeli (Tischbein, 1853)

Nomenclature:

Nematus wesmaeli Tischbein, 1853

Distribution: England, Scotland, Wales, Ireland

\section{Subgenus Lygaeophora Liston, 1993}

Nomenclature:

LYGAEOPHORA Lindqvist, 1952 unavailable name

\section{Pristiphora (Lygaeophora) sermola Liston, 1993}

\section{Nomenclature:}

variipes (Lindqvist, 1952, Lygaeonematus) preocc.

lanifica (Zaddach, 1883): Liston, 1981a misident.

\section{Distribution: Scotland}

Notes: Added by Liston (1981a).

Lygaeophora variipes Lindqvist, 1952 is preoccupied in Pristiphora by $P$. varipes Serville, 1823. The names varipes and variipes are to be regarded as identical spellings: Article 58.14. (ICZN 1999). Pristiphora varipes Serville, 1823 is a species inquirenda, not a synonym of Cladius (Priophorus) pallipes Serville, 1823 or C. brullei (Dahlbom, 1835) (see Lacourt 2000). The conditions of Article 23.9., permitting continued use of the junior homonym as valid, are not fulfilled for variipes (Lindqvist) because this has only been used as a valid name on twenty occasions in works published within the last 50 years (23.9.1.2.).

Subgenus Lygaeotus Liston, 1993

Nomenclature:

LYGAEOTUS Lindqvist, 1952 unavailable name

\section{Pristiphora (Lygaeotus) albilabris (Boheman, 1852)}

Nomenclature:

Nematus albilabris Boheman, 1852

albilabris (Thomson, 1863, Nematus) preocc.

Distribution: England 
Pristiphora (Lygaeotus) borea (Konow, 1904)

Nomenclature:

Lygaeonematus boreus Konow, 1904

astuta (Cameron, 1885, Nematus) nom. ob.

lapponica (Enslin, 1916, Pachynematus)

Distribution: Scotland

Notes: Taxonomy follows Liston et al. (2006).

Pristiphora (Lygaeotus) breadalbanensis (Cameron, 1882)

Nomenclature:

Nematus breadalbanensis Cameron, 1882

tromsoensis (Kiaer, 1898, Lygaeonematus)

corpulentus (Konow, 1904, Lygaeonematus)

arcticola (Enslin, 1916, Lygaeonematus)

Distribution: England, Scotland, Wales, Ireland

\section{Pristiphora (Lygaeotus) carinata (Hartig, 1837)}

Nomenclature:

Nematus carinatus Hartig, 1837

pallipes (Fallén, 1808, Tenthredo) preocc.

Distribution: England, Scotland, Wales

Pristiphora (Lygaeotus) coactula (Ruthe, 1859)

Nomenclature:

Nematus coactulus Ruthe, 1859

alpina (Thomson, 1871, Nematus)

pachyvalvis (Konow, 1904, Lygaeonematus)

Distribution: Scotland

Pristiphora (Lygaeotus) groenblomi (Lindqvist, 1952)

Nomenclature:

Lygaeonematus grönblomi Lindqvist, 1952

gronblomi: misspelling 
Distribution: Scotland

Notes: Predominant usage is of this spelling, not gronblomi, which would be correct according to ICZN Article 32.5.2 (ICZN 1999). Following ICZN (Article 33.3.1) the use of the predominant spelling is maintained.

Pristiphora (Lygaeotus) lativentris (Thomson, 1871)

Nomenclature:

Nematus lativentris Thomson, 1871

scotica (Cameron, 1881, Nematus)

extrema (Holmgren, 1883, Nematus)

Distribution: England, Scotland

Pristiphora (Lygaeotus) mollis (Hartig, 1837)

Nomenclature:

Nematus mollis Hartig, 1837

whitei (Cameron, 1878, Nematus)

kontkaneni (Lindqvist, 1960, Pachynematus)

Distribution: England, Scotland, Wales, Ireland

Pristiphora (Lygaeotus) pseudocoactula (Lindqvist, 1952)

Nomenclature:

Lygaeonematus pseudocoactulus Lindqvist, 1952

pachyvalvis (Konow, 1904): Benson, 1934 misident.

Distribution: England, Scotland, Wales

Subgenus Micronematus Konow, 1890

Nomenclature:

GYMNONYCHUS Marlatt, 1896

Pristiphora (Micronematus) abbreviata (Hartig, 1837)

Nomenclature:

Nematus abbreviatus Hartig, 1837

californica (Marlatt, 1896, Gymnonychus)

Distribution: England 
Pristiphora (Micronematus) biscalis (Förster, 1854)

Nomenclature:

Nematus biscalis Förster, 1854

Distribution: England, Wales, Ireland

Pristiphora (Micronematus) maesta (Zaddach, 1876)

Nomenclature:

Nematus maestus Zaddach, 1876

brevicornis (Thomson, 1862, Nematus) preocc.

parvicornis (Kirby, 1882, Nematus)

insularis (Malaise, 1921, Pachynematus)

moesta: misspelling

Distribution: England

Pristiphora (Micronematus) monogyniae (Hartig, 1840)

Nomenclature:

Nematus monogyniae Hartig, 1840

hibernica (Cameron, 1878, Nematus)

Distribution: England, Wales, Ireland

Pristiphora (Micronematus) retusa (Thomson, 1871)

Nomenclature:

Nematus retusus Thomson, 1871

Distribution: Scotland

Subgenus Oligonematus Zhelochovtsev, 1988

Pristiphora (Oligonematus) friesei (Konow, 1904)

Nomenclature:

Lygaeonematus friesei Konow, 1904

funerula (Costa, 1859): misident.

Distribution: Scotland, Ireland

Notes: Added by O'Connor and Liston (1994). 
Pristiphora (Oligonematus) laricis (Hartig, 1837)

Nomenclature:

Nematus laricis Hartig, 1837

leucocnemis (Förster, 1854, Nematus)

oblonga (Cameron, 1882, Nematus)

laricivora (Brischke, 1883, Nematus)

ravida (Konow, 1903, Pachynematus)

Distribution: England, Scotland, Wales, Ireland

Subgenus Pristiphora Latreille, 1810

Nomenclature:

DIPHADNUS Hartig, 1837

Pristiphora (Pristiphora) alpestris (Konow, 1903)

Nomenclature:

Lygaeonematus alpestris Konow, 1903

Lygaeonematus strandi Konow, 1901: Benson, 1934 misident.

Distribution: England

Pristiphora (Pristiphora) aphantoneura (Förster, 1854)

Nomenclature:

Nematus aphantoneurus Förster, 1854

fulvipes (Fallén, 1808, Tenthredo) preocc.

vicina Serville, 1823: misident.

Distribution: England, Wales

Notes: See Vikberg (2006) on taxonomy.

Pristiphora (Pristiphora) appendiculata (Hartig, 1837)

Nomenclature:

Nematus appendiculatus Hartig, 1837

pallipes Serville, 1823, preocc.

grossulariae Walsh, 1866

rufipes Serville, 1823: misident. 
Distribution: England, Scotland, Ireland

Pristiphora (Pristiphora) armata (Thomson, 1862)

Nomenclature:

Nematus armatus Thomson, 1862

crassicornis (Hartig, 1837, Nematus) preocc.

fletcheri (Cameron, 1884, Nematus)

nigricollis (Cameron, 1885, Nematus)

Distribution: England, Scotland, Wales, Ireland

Pristiphora (Pristiphora) bifida (Hellén, 1948)

Nomenclature:

Nematus bifidus Hellén, 1948

Distribution: Scotland

Pristiphora (Pristiphora) brevis (Hartig, 1837)

Nomenclature:

Nematus brevis Hartig, 1837

fumipennis (Thomson, 1871, Nematus)

fuscata Benson, 1943

thalictri (Kriechbaumer, 1884): misident.

Distribution: England, Ireland

Pristiphora (Pristiphora) cincta Newman, 1837

Nomenclature:

quercus (Hartig, 1837, Nematus)

Distribution: England, Scotland, Wales, Ireland

Notes: Possibly two British species occur under this name: one mainly in the lowlands with hostplant Betula, the other montane with host Vaccinium. Whilst $P$. cincta seems to be the correct name for the more brightly coloured lowland taxon, until a revision is made of the numerous supposed synonyms of $P$. cincta, the name for the species on Vaccinium is unclear. 
Pristiphora (Pristiphora) confusa Lindqvist, 1955

Distribution: England, Scotland, Wales

Pristiphora (Pristiphora) coniceps Lindqvist, 1955

Distribution: England, Scotland

Pristiphora (Pristiphora) conjugata (Dahlbom, 1835)

Nomenclature:

Nematus conjugatus Dahlbom, 1835

Distribution: England

Pristiphora (Pristiphora) denudata Konow, 1902

Nomenclature:

atlantica Lacourt, 1987: misident.

Distribution: England, Scotland, Wales, Ireland

Notes: Contrary to the opinion of Lacourt (1987), we do not consider this taxon to be synonymous with $P$. pallidiventris (Fallén, 1808) ( $P$. pallidiventris pallidiventris sensu Lacourt), nor with $P$. atlantica Malaise, 1939 (= P. atlantica Lacourt, 1987) as previously treated by Liston (1995), who replaced the name denudata with atlantica Lacourt on the grounds that the name denudata is preoccupied in Pristiphora by Nematus denudatus Hartig, 1840. Nematus denudatus was at that time considered to be a synonym of Pristiphora carinata (Hartig, 1837). According to Schmidt (in Lacourt 1999), N. denudatus Hartig actually belongs to Amauronematus (Brachycoluma), thus removing the homonymy. $P$. denudata differs from $P$. pallidiventris not only in the more extensively pale abdomen (without intermediate specimens), but also in details of the structure of the saw, and is probably attached only to Rubus species, whereas $P$. pallidiventris is polyphagous on various Rosaceae.

Pristiphora (Pristiphora) geniculata (Hartig, 1840)

Nomenclature:

Nematus geniculatus Hartig, 1840

Distribution: England, Ireland

Pristiphora (Pristiphora) insularis Rohwer, 1910

Nomenclature:

kamtchatica Malaise, 1931 
mesatlantica Lacourt, 1976

luteiventris Koch, 1989

paedida (Konow, 1904): misident.

Distribution: England, Scotland, Wales, Ireland

Notes: Synonymy follows Haris (2006).

Pristiphora (Pristiphora) leucopus (Hellén, 1948)

Nomenclature:

Nematus leucopus Hellén, 1948

Distribution: England, Scotland

Notes: Added by Grearson (2006).

Pristiphora (Pristiphora) luteipes Lindqvist, 1955

Nomenclature:

pygmaea Lindqvist, 1964

Distribution: England, Scotland, Wales, Ireland

Notes: Added by O'Connor et al. (1997).

See Vikberg (2006) on taxonomy.

Pristiphora (Pristiphora) melanocarpa (Hartig, 1840)

Nomenclature:

Nematus melanocarpus Hartig, 1840

wuestneii (R. Stein, 1885, Nematus)

Distribution: England, Scotland, Wales, Ireland

Pristiphora (Pristiphora) pallidiventris (Fallén, 1808)

Nomenclature:

Tenthredo pallidiventris Fallén, 1808

ephippiger (Hartig, 1840, Nematus)

flavicoma (Tischbein, 1840, Nematus)

nigricans (Eversmann, 1847, Nematus)

caudalis (Eversmann, 1847, Nematus) 
breviuscula (Eversmann, 1847, Nematus)

gemella (Förster, 1854, Nematus)

cirrhostoma (Zaddach, 1883, Nematus)

Pristiphora myosotidis (Fabricius, 1804): Stephens, 1835 misident.

Nematus obductus (Hartig, 1837): Cameron, 1874 misident.

Distribution: England, Scotland, Wales, Ireland

\section{Pristiphora (Pristiphora) punctifrons (Thomson, 1871)}

Nomenclature:

Nematus punctifrons Thomson, 1871

Distribution: England, Scotland, Wales, Ireland

\section{Pristiphora (Pristiphora) ruficornis (Olivier, 1811)}

Nomenclature:

Nematus ruficornis Olivier, 1811

fraxini (Hartig, 1837, Nematus)

Distribution: England, Scotland, Wales, Ireland

Pristiphora (Pristiphora) rufipes Serville, 1823

Nomenclature:

aquilegiae (Vollenhoven, 1866, Nematus)

alnivora (Hartig, 1840): misident.

Distribution: England, Scotland

\section{Pristiphora (Pristiphora) staudingeri (Ruthe, 1859)}

Nomenclature:

Nematus staudingeri Ruthe, 1859

circularis Kincaid, 1900

?hyperborea Malaise, 1921

asperlatus Benson, 1935

Distribution: England, Scotland, Wales, Ireland

Notes: Pristiphora hyperborea was recorded as British by Benson (1935), but later treated by him as a synonym of $P$. staudingeri. V. Vikberg considers $P$. hyperborea to 
represent a valid species (see Zhelochovtsev 1988). Probably both species occur in the British Isles.

\section{Pristiphora (Pristiphora) subbifida (Thomson, 1871)}

Nomenclature:

Nematus subbifidus Thomson, 1871

Distribution: England, Wales

Notes: Characterisation of $P$. subbifida by Zhelochovtsev (1988) is based on misidentification by Lindqvist (1973) of $P$. conjugata, or a similar species.

\section{Pristiphora (Pristiphora) subopaca Lindqvist, 1955}

Distribution: England

Notes: Recorded by Lindqvist (1955) from Great Britain, but in the reprints of this paper which he distributed, "Grossbritannien" is scored out. Therefore this record is considered to be based on a misidentification. However, J. Grearson has recently taken females in England, identity confirmed by V. Vikberg after comparison with the holotype (Grearson, in preparation).

Pristiphora (Pristiphora) testacea (Jurine, 1807)

Nomenclature:

Pteronus testaceus Jurine, 1807

betulae (Retzius, 1783, Tenthredo) preocc.

crassiventris (Cameron, 1878, Nematus)

Distribution: England, Scotland, Wales, Ireland

Pristiphora (Pristiphora) thalictri (Kriechbaumer, 1884)

Nomenclature:

Nematus thalictri Kriechbaumer, 1884

Distribution: Scotland

Notes: Added by Liston et al. (2012).

Subgenus Sharliphora Wong, 1969 
Pristiphora (Sharliphora) amphibola (Förster, 1854)

Nomenclature:

Nematus amphibolus Förster, 1854

laeta (Cameron, 1883, Nematus)

fraterna (Cameron, 1885, Nematus)

Distribution: England, Scotland, Wales

Pristiphora (Sharliphora) nigella (Förster, 1854)

Nomenclature:

Nematus nigellus Förster, 1854

ambigua (Fallén, 1808, Tenthredo) preocc.

furvescens (Cameron, 1876, Nematus)

Distribution: England, Scotland, Ireland

Tribe Pseudodineurini Benson, 1938

Genus Endophytus Hering, 1934

Nomenclature:

NEOPELMATOPUS Conde, 1934

\section{Endophytus anemones (Hering, 1924)}

Nomenclature:

Pelmatopus anemones Hering, 1924

tenuiserra (Lindqvist, 1949, Pelmatopus)

Distribution: England

\section{Genus Pseudodineura Konow, 1885}

Nomenclature:

PELMATOPUS Hartig, 1837 preocc.

PHYLLOPAIS Hering, 1934

Pseudodineura enslini (Hering, 1923)

Nomenclature:

Pelmatopus enslini Hering, 1923 
Distribution: England, Scotland, Wales

Pseudodineura fuscula (Klug, 1816)

Nomenclature:

Tenthredo fuscula Klug, 1816

despecta (Hartig, 1837, Dineura)

simulans (Cameron, 1877, Dineura)

Distribution: England, Scotland, Ireland

Pseudodineura mentiens (Thomson, 1871)

Nomenclature:

Blennocampa mentiens Thomson, 1871

Distribution: Scotland

Notes: Added by Liston and Blank (2006).

Tribe Stauronematini Lacourt, 1998

Genus Stauronematus Benson, 1953

Nomenclature:

STAURONEMA Benson, 1948 preocc.

Stauronematus platycerus (Hartig, 1840)

Nomenclature:

Nematus platycerus Hartig, 1840

compressicornis (Fabricius, 1804): misident.

Distribution: England, Scotland, Wales

Subfamily Selandriinae Thomson, 1871

Tribe Aneugmenini Takeuchi, 1941

Genus Aneugmenus Hartig, 1837

Nomenclature:

ATOPOSELANDRIA Enslin, 1913 


\section{Aneugmenus coronatus (Klug, 1818)}

Nomenclature:

Tenthredo coronata Klug, 1818

analis (Thomson, 1871, Selandria)

cereipes (Vollenhoven, 1873, Selandria)

Distribution: England, Scotland, Ireland

Notes: Not a synonym of $A$. padi. See Chevin (1980). There is a major divergence in opinion regarding the association of the sexes of these species: compare, for example, Blank (1998), Chevin (1980) and Benson (1952), Benson (1968).

\section{Aneugmenus fuerstenbergensis (Konow, 1885)}

Nomenclature:

Selandria fuerstenbergensis Konow, 1885

furstenbergensis: misspelling

Distribution: England, Scotland, Wales, Ireland

\section{Aneugmenus padi (Linnaeus, 1760)}

Nomenclature:

Tenthredo padi Linnaeus, 1760

stramineipes (Klug, 1816, Tenthredo)

Distribution: England, Scotland, Wales, Ireland

\section{Aneugmenus temporalis (Thomson, 1871)}

Nomenclature:

Selandria temporalis Thomson, 1871

Distribution: England, Scotland, Wales, Ireland

Tribe Dolerini Thomson, 1871

Genus Dolerus Panzer, 1801

\section{Subgenus Achaetoprion Goulet, 1986}

Nomenclature: 
Dolerus (Achaetoprion) ferrugatus Serville, 1823

Distribution: England, Scotland, Wales, Ireland

Dolerus (Achaetoprion) madidus (Klug, 1818)

Nomenclature:

Tenthredo madida Klug, 1818

lateritius (Klug, 1818, Tenthredo)

chappelli Cameron, 1877

schulthessi Konow, 1887: ?misident.

Distribution: England, Scotland, Wales, Ireland

Dolerus (Achaetoprion) pachycerus Hartig, 1837

Nomenclature:

taeniatus Zaddach, 1859

tinctipennis Cameron, 1881

Distribution: England, Scotland, Wales

Dolerus (Achaetoprion) triplicatus (Klug, 1818)

Nomenclature:

Tenthredo triplicata Klug, 1818

steini Konow, 1885

Distribution: England, Wales

Subgenus Cyperolerus Zhelochovtsev, 1988

Dolerus (Cyperolerus) anticus (Klug, 1818)

Nomenclature:

Tenthredo antica Klug, 1818

Distribution: England

Subgenus Dicrodolerus Goulet, 1986

Nomenclature:

Tenthredo vestigialis Klug, 1818 
Dolerus (Dicrodolerus) vestigialis (Klug, 1818)

Nomenclature:

Tenthredo vestigialis Klug, 1818

genucinctus Zaddach, 1859: Benson, 1934 misident.

Loderus vestigialis: Benson, 1952

Distribution: England, Scotland, Wales, Ireland

Subgenus Dolerus Panzer, 1801

Nomenclature:

DOLERUS Jurine, 1801 suppressed

DOSYTHEUS Leach, 1817

Dolerus (Dolerus) aericeps Thomson, 1871

Nomenclature:

bajulus Serville, 1823 nom. ob.

Distribution: England, Scotland, Wales, Ireland

Dolerus (Dolerus) bimaculatus (Geoffroy, 1785)

Nomenclature:

Tenthredo bimaculata Geoffroy, 1785

tristis (Fabricius, 1804, Tenthredo) preocc.

Distribution: England, Scotland, Wales, Ireland

Dolerus (Dolerus) cothurnatus Serville, 1823

Nomenclature:

palustris (Klug, 1818, Tenthredo) preocc.

junci (Stephens, 1835, Dosytheus)

Distribution: England, Scotland, Wales, Ireland

Dolerus (Dolerus) germanicus (Fabricius, 1775)

Nomenclature:

Tenthredo germanica Fabricius, 1775

fuscipennis (Stephens, 1835, Dosytheus)

fulviventris (Scopoli, 1763): Cameron, 1882 misident. 
pratensis (Linnaeus, 1758): misident.

Distribution: England, Scotland, Wales, Ireland

Dolerus (Dolerus) gessneri André, 1880

Nomenclature:

labiosus Konow, 1897

Distribution: England, Scotland, Wales, Ireland

Dolerus (Dolerus) yukonensis Norton, 1872

Nomenclature:

scoticus Cameron, 1881

lateralis Konow, 1895

arcticola Kiaer, 1898

saxatilis Hartig, 1837: Benson, 1952 misident.

Distribution: England, Scotland, Wales

Notes: Haris (2000) regards D. scoticus as a species distinct from D. yukonensis (= arcticola Kiaer). Benson (1935) had already drawn essentially the same conclusions.

D. scoticus was subsequently (Benson 1952) regarded as a synonym of Dolerus saxatilis and latterly (Benson 1958: corrigenda) as a synonym of $D$. yukonensis. Goulet (1986) concurred with the synonymy of $D$. scoticus and $D$. yukonensis.

\section{Subgenus Equidolerus Taeger \& Blank, 1996}

Nomenclature:

DOSYTHEUS: Goulet, 1986 misident.

\section{Dolerus (Equidolerus) pratensis (Linnaeus, 1758)}

Nomenclature:

Tenthredo pratensis Linnaeus, 1758

dubius (Klug, 1818, Tenthredo)

timidus (Klug, 1818, Tenthredo)

Distribution: England, Scotland, Wales, Ireland 
Subgenus Loderus Konow, 1890

Dolerus (Loderus) gilvipes (Klug, 1818)

Nomenclature:

Tenthredo gilvipes Klug, 1818

pratorum ssp. gilvipes (Klug, 1818)

Distribution: England, Scotland

Dolerus (Loderus) pratorum (Fallén, 1808)

Nomenclature:

Tenthredo pratorum Fallén, 1808

Distribution: England, Wales

Subgenus Oncodolerus Goulet, 1986

Dolerus (Oncodolerus) eversmanni Kirby, 1882

Nomenclature:

palmatus (Klug, 1818, Tenthredo) preocc.

Loderus eversmanni: Benson, 1952

Distribution: England, Scotland, Wales, Ireland

Subgenus Poodolerus Zhelochovtsev, 1988

Dolerus (Poodolerus) aeneus Hartig, 1837

Nomenclature:

elongatus Thomson, 1871

Distribution: England, Scotland, Wales, Ireland

Dolerus (Poodolerus) anthracinus (Klug, 1818)

Nomenclature:

Tenthredo anthracina Klug, 1818

Distribution: England, Scotland, Wales 


\section{Dolerus (Poodolerus) asper Zaddach, 1859}

Nomenclature:

carbonarius Zaddach, 1859

oblongus Cameron, 1882

planatus Hartig, 1837: misident.

Distribution: England, Scotland

Notes: Previous records of $D$. asper from Wales and Ireland require checking, because they may refer to $D$. brevicornis (below), recently distinguished by Heidemaa et al. (2004). English specimens of $D$. asper, leg. K. J. Grearson, have been determined by M. Heidemaa. The only evidence for the presence of $D$. asper in Scotland is the determination of a syntype specimen of Dolerus oblongus in NHM, examined by Heidemaa et al. (2004) and identified as D. asper. Cameron (1882) described $D$. oblongus from "Braemar, Rannoch, Clydesdale".

\section{Dolerus (Poodolerus) brevicornis Zaddach, 1859}

Nomenclature:

asper Zaddach, 1859: misident.

Distribution: England, Scotland

Notes: Only recently distinguished from $D$. asper (see above). A specimen collected by K. J. Grearson in England has been determined as D. brevicornis by M. Heidemaa.

\section{Dolerus (Poodolerus) coracinus (Klug, 1818)}

Nomenclature:

Tenthredo coracina Klug, 1818

Distribution: England, Scotland

\section{Dolerus (Poodolerus) fumosus Stephens, 1835}

\section{Nomenclature:}

sanguinicollis (Klug, 1818): misident.

Distribution: England, Scotland, Wales

\section{Dolerus (Poodolerus) gonager (Fabricius, 1781)}

Nomenclature:

Tenthredo gonagra Fabricius, 1781 
Distribution: England, Scotland, Wales

Dolerus (Poodolerus) haematodes (Schrank, 1781)

Nomenclature:

Tenthredo haematodes Schrank, 1781

collaris (Donovan, 1808, Tenthredo)

Distribution: England, Scotland, Wales, Ireland

Dolerus (Poodolerus) harwoodi Benson, 1947

Distribution: Scotland

Dolerus (Poodolerus) liogaster Thomson, 1871

Distribution: England, Scotland, Wales, Ireland

Notes: Heidemaa (2004) established that D. schmidti (below) has hitherto been confused with $D$. liogaster. $D$. liogaster is confirmed as present in Britain by $M$. Heidemaa (pers. comm.) based on material collected by A. Barker.

Dolerus (Poodolerus) niger (Linnaeus, 1767)

Nomenclature:

Tenthredo nigra Linnaeus, 1767

Distribution: England, Scotland, Wales, Ireland

Dolerus (Poodolerus) nigratus (Müller, 1776)

Nomenclature:

Tenthredo nigrata Müller, 1776

fissus Hartig, 1837

Distribution: England, Scotland, Wales, Ireland

Dolerus (Poodolerus) nitens Zaddach, 1859

Nomenclature:

coruscans Konow, 1890

wanda Ross, 1935

Distribution: England, Scotland, Wales, Ireland 
Dolerus (Poodolerus) picipes (Klug, 1818)

Nomenclature:

Tenthredo picipes Klug, 1818

intermedius Cameron, 1881

Distribution: England, Scotland, Wales, Ireland

Dolerus (Poodolerus) possilensis Cameron, 1882

Nomenclature:

nitens Zaddach, 1859: misident.

Distribution: England, Scotland, Wales, Ireland

Dolerus (Poodolerus) puncticollis Thomson, 1871

Distribution: England, Scotland, Wales, Ireland

\section{Dolerus (Poodolerus) schmidti Konow, 1884}

Distribution: England, Scotland

Notes: See under D. liogaster (above). D. schmidti is present in Britain according to determinations made by M. Heidemaa: England, leg. K. J. Grearson and Scotland, leg.

A. Barker.

Dolerus (Poodolerus) stygius Förster, 1860

Nomenclature:

megapterus Cameron, 1881

Distribution: England, Scotland, Wales

Dolerus (Poodolerus) varispinus Hartig, 1837

Nomenclature:

rugosus Konow, 1884

rugosulus Dalla Torre, 1894

brevitarsis: Benson, 1947 misident. and misspelling

liogaster Thomson, 1871: misident.

Distribution: England, Scotland, Wales, Ireland

Notes: Taxonomy follows Heidemaa (2004). 
Tribe Dulophanini Lacourt, 1998

Genus Birka Malaise, 1944

Birka cinereipes (Klug, 1816)

Nomenclature:

Tenthredo cinereipes Klug, 1816

aperta (Hartig, 1837, Tenthredo)

Distribution: England, Scotland, Wales, Ireland

Genus Nesoselandria Rohwer, 1910

Nomenclature:

MELISANDRA Benson, 1939

DULOPHANES Konow, 1907: misident.

Nesoselandria morio (Fabricius, 1781)

Nomenclature:

Tenthredo morio Fabricius, 1781

Distribution: England, Scotland, Wales, Ireland

Tribe Selandriini Thomson, 1871

Genus Brachythops Curtis, 1839

Nomenclature:

CORYNA Lepeletier \& Serville, 1825 preocc.

Brachythops flavens (Klug, 1816)

Nomenclature:

Tenthredo flavens Klug, 1816

scapularis (Lepeletier \& Serville, 1828, Tenthredo)

seminigra Curtis, 1839

flavescens (Thomson, 1870, Selandria)

Distribution: England, Scotland, Wales, Ireland 
Brachythops wuestneii (Konow, 1885)

Nomenclature:

Selandria wuestneii Konow 1885

flavistigma (Groenblom, 1939, Selandria)

Distribution: England, Scotland, Wales

\section{Genus Selandria Leach, 1817}

Selandria melanosterna (Serville, 1823)

Nomenclature:

Tenthredo melanosterna Serville, 1823

sixii Vollenhoven, 1858

grandis Zaddach, 1859

interstitialis Thomson, 1871

dorsalis Stephens, 1835: Kirby, 1882 misident.

Distribution: England, Scotland, Wales

Selandria serva (Fabricius, 1793)

Nomenclature:

Tenthredo serva Fabricius, 1793

dorsalis Stephens, 1835

excisa Konow, 1885

fuscitarsis Benson, 1954

Distribution: England, Scotland, Wales, Ireland

Tribe Strongylogastrini Ashmead, 1898

Genus Pseudohemitaxonus Conde, 1932

Pseudohemitaxonus sharpi (Cameron, 1879)

Nomenclature:

Strongylogaster sharpi Cameron, 1879

exsectus Conde, 1932

mixtus (Klug, 1817): Cameron, 1874 misident. 
Strongylogaster contigua (Konow, 1885): misident.

Distribution: England, Scotland

Genus Stromboceros Konow, 1885

Nomenclature:

STROMBOCERINA Malaise, 1942

STROMBOCERUS: misspelling

Stromboceros delicatulus (Fallén, 1808)

Nomenclature:

Tenthredo delicatula Fallén, 1808

Strongylogaster eborina (Klug, 1817, Tenthredo)

Selandria phthisica Vollenhoven, 1869

Distribution: England, Scotland, Wales, Ireland

Genus Strongylogaster Dahlbom, 1835

Nomenclature:

PSEUDOTAXONUS Costa, 1894

\section{Strongylogaster filicis (Klug, 1817)}

Nomenclature:

Tenthredo filicis Klug, 1817

Distribution: †England

Notes: Extinct; recorded only on the basis of a single specimen by Bold (1873). The present whereabouts of the specimen are unknown.

\section{Strongylogaster macula (Klug, 1817)}

Nomenclature:

Tenthredo macula Klug, 1817

intermedia (Konow, 1885, Thrinax)

mixtus (Klug, 1817): Cameron, 1874 misident. and misspelling

Distribution: England, Scotland, Wales, Ireland 
Strongylogaster mixta (Klug, 1817)

Nomenclature:

Tenthredo mixta Klug, 1817

femoralis Cameron, 1875

Distribution: England, Scotland, Wales, Ireland

Strongylogaster multifasciata (Geoffroy, 1785)

Nomenclature:

Tenthredo multifasciata Geoffroy, 1785

lineata (Christ, 1791, Tenthredo)

atricornis (Stephens, 1835, Tenthredo)

cingulata (Fabricius, 1793): misident.

Distribution: England, Scotland, Wales, Ireland

Strongylogaster xanthocera (Stephens, 1835)

Nomenclature:

Tenthredo xanthocera Stephens, 1829

geniculata Thomson, 1871

xanthoceros: misspelling

Distribution: England, Scotland, Wales

Subfamily Tenthredininae Latreille, 1802

Tribe Macrophyini Benson, 1946

Genus Macrophya Dahlbom, 1835

Nomenclature:

PSEUDOMACROPHYA Enslin, 1913

Macrophya albicincta (Schrank, 1776)

Nomenclature:

Tenthredo albicincta Schrank, 1776

Distribution: England, Scotland, Wales 
Macrophya albipuncta (Fallén, 1808)

Nomenclature:

Tenthredo albipuncta Fallén, 1808

Distribution: England, Scotland

Macrophya alboannulata Costa, 1859

Distribution: England, Wales

Notes: Added by Liston (1983a).

Macrophya annulata (Geoffroy, 1785)

Nomenclature:

Tenthredo annulata Geoffroy, 1785

neglecta (Klug, 1817, Tenthredo)

Distribution: England, Wales

Macrophya blanda (Fabricius, 1775)

Nomenclature:

Tenthredo blanda Fabricius, 1775

Distribution: England

Macrophya duodecimpunctata (Linnaeus, 1758)

Nomenclature:

Tenthredo duodecimpunctata Linnaeus, 1758

Distribution: England, Scotland, Wales, Ireland

Macrophya montana (Scopoli, 1763)

Nomenclature:

Tenthredo montana Scopoli, 1763

rustica (Linnaeus, 1758): misident.

Distribution: England, Scotland, Wales 
Macrophya punctumalbum (Linnaeus, 1767)

Nomenclature:

Tenthredo punctumalbum Linnaeus, 1767

erythropus (Schrank, 1776, Tenthredo)

parvula Konow, 1884: Liston, 1987 misident.

Distribution: England, Wales, Ireland

Macrophya ribis (Schrank, 1781)

Nomenclature:

Tenthredo ribis Schrank, 1781

Distribution: England, Wales

Macrophya rufipes (Linnaeus, 1758)

Nomenclature:

Tenthredo rufipes Linnaeus, 1758

ione (Newman, 1837, Allantus)

haematopus (Panzer, 1801): Cameron, 1882 misident.

Distribution: England, Wales

Genus Pachyprotasis Hartig, 1837

Pachyprotasis antennata (Klug, 1817)

Nomenclature:

Tenthredo antennata Klug, 1817

Distribution: England, Scotland, Wales, Ireland

Pachyprotasis nigronotata Kriechbaumer, 1874

Distribution: Wales

Pachyprotasis rapae (Linnaeus, 1767)

Nomenclature:

Tenthredo rapae Linnaeus, 1767

Distribution: England, Scotland, Wales, Ireland 
Pachyprotasis simulans (Klug, 1817)

Nomenclature:

Tenthredo simulans Klug, 1817

Distribution: England, Scotland, Wales

Pachyprotasis variegata (Fallén, 1808)

Nomenclature:

Tenthredo variegata Fallén, 1808

Distribution: England, Scotland, Wales

Tribe Perineurini Rohwer, 1911

Genus Aglaostigma W. F. Kirby, 1882

Aglaostigma aucupariae (Klug, 1817)

Nomenclature:

Tenthredo aucupariae Klug, 1817

solitaria (Fallén, 1808, Tenthredo) preocc.

juvenilis (Serville, 1823, Tenthredo)

Tenthredo gibbosa Fallén, 1808: Thomson, 1870 misident.

Distribution: England, Scotland, Wales, Ireland

Aglaostigma fulvipes (Scopoli, 1763)

Nomenclature:

Tenthredo fulvipes Scopoli, 1763

lateralis (Fabricius, 1779, Tenthredo)

Distribution: England, Scotland, Wales, Ireland

Genus Perineura Hartig, 1837

Nomenclature:

SYNAIREMA Hartig, 1837 
Perineura rubi (Panzer, 1803)

Nomenclature:

Allantus rubi Panzer, 1803

Distribution: England, Scotland, Wales, Ireland

Genus Tenthredopsis Costa, 1859

Nomenclature:

EBOLIA Costa, 1859

THOMSONIA Konow, 1884

EUTENTHREDOPSIS Enslin, 1913

Tenthredopsis coquebertii (Klug, 1817)

Nomenclature:

Tenthredo coquebertii Klug, 1817

nigricollis Cameron, 1881

opulenta Konow, 1887

ignobilis (Klug, 1817): Cameron, 1882 misident.

Distribution: England, Scotland, Wales, Ireland

\section{Tenthredopsis friesei (Konow, 1884)}

Nomenclature:

Thomsonia friesei Konow, 1884

?palmata (Geoffroy, 1785, Tenthredo)

laticeps (Konow, 1884, Thomsonia)

korlevici Konow, 1887

arrogans Konow, 1890

pavida (Fabricius, 1775): misident.

Distribution: England, Scotland

Tenthredopsis litterata (Geoffroy, 1785)

Nomenclature:

Tenthredo litterata Geoffroy, 1785

carbonaria (Linnaeus, 1767, Tenthredo) preocc.

cordata (Geoffroy, 1785, Tenthredo) 
microcephala (Serville, 1823, Tenthredo)

caliginosa (Stephens, 1835, Tenthredo)

femoralis (Stephens, 1835, Tenthredo)

nigriceps Cameron, 1881

nigronotata Cameron, 1881

pallida Konow, 1896

Distribution: England, Scotland, Wales

Tenthredopsis nassata (Linnaeus, 1767)

Nomenclature:

Tenthredo nassata Linnaeus, 1767

alneti (Schrank, 1781, Tenthredo)

dorsalis (Serville, 1823, Tenthredo)

fulviceps (Stephens, 1835, Tenthredo)

tristis (Stephens, 1835, Tenthredo)

albomaculata Cameron, 1881

dorsivittata Cameron, 1881

inornata Cameron, 1881

lividiventris Cameron, 1881

saundersi Cameron, 1881

elegans (Konow, 1884, Thomsonia)

gibberosa Konow, 1887

fenestrata Konow, 1890

tristior Morice, 1914

sordida (Klug, 1817): misident.

Distribution: England, Scotland, Wales, Ireland

\section{Tenthredopsis ornata (Serville, 1823)}

Nomenclature:

Tenthredo ornata Serville, 1823

excisa (Thomson, 1870, Perineura)

tesselata: Benson, 1968 misident. and misspelling

Distribution: England, Scotland 
Tenthredopsis scutellaris (Fabricius, 1804)

Nomenclature:

Tenthredo scutellaris Fabricius, 1804

spreta (Serville, 1823, Tenthredo)

picticeps Cameron, 1881

flavomaculata Cameron, 1881

austriaca Konow, 1890

dubia Konow, 1890

parvula Konow, 1890

puncticollis Konow, 1890

thornleyi Konow, 1899

nassata: misident.

campestris (Linnaeus, 1758): misident.

Distribution: England, Scotland, Ireland

Tribe Tenthredinini Latreille, 1802

Nomenclature:

SCIAPTERYGINI Benson, 1946

Genus Cytisogaster Lacourt, 1996

Cytisogaster chambersi (Benson, 1947)

Nomenclature:

Rhogogaster chambersi Benson, 1947

Distribution: England, Scotland, Wales

Cytisogaster genistae (Benson, 1947)

Nomenclature:

Rhogogaster genistae Benson, 1947

Distribution: England, Scotland, Wales

Cytisogaster picta (Klug, 1817)

Nomenclature:

Tenthredo picta Klug, 1817 
Distribution: England, Wales

Genus Rhogogaster Konow, 1884

Nomenclature:

RHOGOGASTERA Konow, 1885

Rhogogaster chlorosoma (Benson, 1943)

Nomenclature:

Tenthredo chlorosoma Benson, 1943

Distribution: England, Scotland, Wales

Rhogogaster dryas (Benson, 1943)

Nomenclature:

Tenthredo dryas Benson, 1943

similis Lindqvist, 1959

Distribution: England, Scotland

\section{Rhogogaster punctulata (Klug, 1817)}

Nomenclature:

Tenthredo punctulata Klug, 1817

Distribution: England, Scotland, Wales

Rhogogaster viridis (Linnaeus, 1758)

Nomenclature:

Tenthredo viridis Linnaeus, 1758

scalaris (Klug, 1817, Tenthredo)

Distribution: England, Scotland, Wales, Ireland

Genus Sciapteryx Stephens, 1835

Nomenclature:

SCIOPTERYX Cameron, 1882: misspelling 
Sciapteryx consobrina (Klug, 1816)

Nomenclature:

Tenthredo consobrina Klug, 1816

Distribution: England

Sciapteryx soror Konow, 1890

Nomenclature:

costalis (Fabricius, 1775): misident.

Distribution: England, Scotland

Genus Tenthredo Linnaeus, 1758

Nomenclature:

TENTHREDELLA Rohwer, 1910

ELINORA Benson, 1940

EUROGASTER Zirngiebl, 1953

CUNEALA Zirngiebl, 1956

CEPHALEDO Zhelochovtsev, 1988

MACULEDO Zhelochovtsev, 1988

MURCIANA Lacourt, 1988

OLIVACEDO Zhelochovtsev, 1988

TEMULEDO Zhelochovtsev, 1988

ZONULEDO Zhelochovtsev, 1988

BLANKIA Lacourt, 1998

Tenthredo amoena Gravenhorst, 1807

Nomenclature:

cingulum Klug, 1817

inversa (Costa, 1894, Allantus)

Distribution: England, Wales

Tenthredo arcuata Forster, 1771

Distribution: England, Scotland, Wales, Ireland 
Tenthredo atra Linnaeus, 1758

Nomenclature:

dispar Klug, 1817

scotica Cameron, 1882

Distribution: England, Scotland, Wales, Ireland

\section{Tenthredo baetica Spinola, 1846}

Nomenclature:

flavipes Geoffroy, 1785: Cameron, 1882 misident.

flaveola Gmelin, 1790: misident.

Distribution: England

Notes: The subspecies baetica dominiquei (Konow, 1894, Allantus) is represented in Britain; this taxon was placed for a time by Benson as a subspecies of the more continental T. flaveola Gmelin, 1790, but his opinion is not supported by data on distribution and morphology. Lacourt (1999) treated T. dominiquei as a northern subspecies of $T$. baetica (nominate subspecies confined to Spain and Portugal). The latter treatment is supported by results of $\mathrm{COI}$ barcoding (unpublished).

\section{Tenthredo balteata Klug, 1817}

Distribution: England, Scotland, Wales, Ireland

\section{Tenthredo brevicornis (Konow, 1886)}

Nomenclature:

Allantus brevicornis Konow, 1886

nitidior (Konow, 1888, Allantus)

aegra Enslin, 1912

acerrima Benson, 1952

sulphuripes (Kriechbaumer, 1869): Benson, 1940 misident.

Distribution: England, Scotland, Wales, Ireland

\section{Tenthredo colon Klug, 1817}

Nomenclature:

nigricollis Kirby, 1882

Distribution: England, Scotland, Wales, Ireland 
Tenthredo distinguenda (R. Stein, 1885)

Nomenclature:

Allantus distinguendus R. Stein, 1885

Distribution: England

Tenthredo fagi Panzer, 1798

Nomenclature:

solitaria Scopoli, 1763: Cameron, 1882 misident.

Distribution: England, Scotland

Tenthredo ferruginea Schrank, 1776

Nomenclature:

rufiventris Panzer, 1799

Distribution: England, Scotland, Wales, Ireland

Tenthredo ignobilis Klug, 1817

Distribution: Scotland

Notes: Added by Liston et al. (2012).

Tenthredo livida Linnaeus, 1758

Distribution: England, Scotland, Wales, Ireland

Tenthredo maculata Geoffroy, 1785

Distribution: England, Scotland, Wales

Tenthredo mandibularis Fabricius, 1804

Distribution: England, Scotland, Wales

Tenthredo mesomela Linnaeus, 1758

Nomenclature:

mesomelas: misspelling

Distribution: England, Scotland, Wales, Ireland 
Tenthredo mioceras (Enslin, 1912)

Nomenclature:

Tenthredella mioceras Enslin, 1912

Distribution: England, Scotland, Ireland

Tenthredo moniliata Klug, 1817

Nomenclature:

lachlaniana Cameron, 1878

Distribution: England, Scotland, Wales, Ireland

Tenthredo neobesa Zombori, 1980

Nomenclature:

pseudorossii Taeger, 1985

rossii (Panzer, 1803): Benson, 1952 misident.

temula Scopoli, 1763: Cameron misident.

vidua Rossi, 1790: Cameron misident.

Distribution: England

Notes: Possibly extinct in Britain.

Tenthredo notha Klug, 1817

Nomenclature:

perkinsi (Morice, 1919, Allantus)

schaefferi Klug, 1817: Benson, 1959 misident.

Distribution: England, Scotland, Wales, Ireland

Tenthredo obsoleta Klug, 1817

Distribution: England, Scotland, Ireland

Tenthredo olivacea Klug, 1817

Distribution: England, Scotland, Wales 
Tenthredo omissa (Förster, 1844)

Nomenclature:

Allantus omissus Förster, 1844

marginella Fabricius, 1793: Thomson, 1871 misident.

Distribution: England

Tenthredo schaefferi Klug, 1817

Distribution: England

Tenthredo scrophulariae Linnaeus, 1758

Distribution: England, Scotland, Wales

Tenthredo semicolon Mol, 2013

Distribution: England

Notes: Added by Knight \& Liston (in prep.).

Tenthredo temula Scopoli, 1763

Nomenclature:

bicincta Linnaeus, 1767

celtica Benson, 1953

Distribution: England, Scotland, Wales, Ireland

Tenthredo thompsoni (Curtis, 1839)

Nomenclature:

Allantus thompsoni Curtis, 1839

marginella Fabricius, 1793: misident.

Distribution: England, Wales

Tenthredo velox Fabricius, 1798

Nomenclature:

velox ab. nigrolineata Cameron, 1878 infrasubspecific name

Distribution: England, Scotland, Wales 
Tenthredo vespa Retzius, 1783

Nomenclature:

multifasciata Geoffroy, 1785: misident.

tricincta Fabricius, 1804

quadricincta Fallén, 1808

Distribution: England, Scotland, Wales

Tenthredo zona Klug, 1817

Nomenclature:

quadricincta Fallén, 1808: Cameron, 1882 misident.

Distribution: England

\section{Superfamily XIPHYDRIOIDEA Leach, 1819}

Family Xiphydriidae Leach, 1819

Subfamily Xiphydriinae Leach, 1819

Genus Xiphydria Latreille, 1802

Nomenclature:

HYBONOTUS Klug, 1803

XIPHIURA Fallén, 1813

XIPHIDRIA Lamarck, 1817

HYPONOTUS Billberg, 1820

XYPHIDRIA Lepeletier, 1823

KONOWIA Brauns, 1884

PSEUDOXIPHYDRIA Enslin, 1911

Xiphydria camelus (Linnaeus, 1758)

Nomenclature:

Ichneumon camelus Linnaeus, 1758

Distribution: England, Scotland, Wales 
Xiphydria longicollis (Geoffroy, 1785)

Nomenclature:

Tenthredo longicollis Geoffroy, 1785

Distribution: England

Notes: Added by Shaw and Liston (1985).

Xiphydria prolongata (Geoffroy, 1785)

Nomenclature:

Tenthredo prolongata Geoffroy, 1785

Sirex dromedarius Fabricius, 1787

Distribution: England, Wales

\section{Superfamily XYELOIDEA Newman, 1834}

Family Xyelidae Newman, 1834

Subfamily Xyelinae Newman, 1834

Tribe Xyelini Newman, 1834

Genus Xyela Dalman, 1819

Nomenclature:

PINICOLA Brébisson, 1818 preocc.

XYELATANA Benson, 1938

Xyela curva Benson, 1938

Distribution: England, Wales

Notes: Added by Liston and Blank (2006).

Xyela julii (Brébisson, 1818)

Nomenclature:

Pinicola julii Brébisson, 1818

Distribution: England, Scotland, Wales, Ireland 


\title{
Xyela longula Dalman, 1819
}

\author{
Nomenclature: \\ piliserra Thomson, 1871
}

Distribution: Scotland

\section{References}

- $\quad$ Altenhofer E, Zombori L (1987) The species of Heterarthrus Stephens, 1835 feeding on maple (Hymenoptera, Tenthredinidae). Annales historico-naturales Musei Nationalis Hungarici 79: 185-197. URL: http://annales.nhmus. hu/cikkreszletes.php?idhoz=1459

- Beneš K, Krístek J (1976) A new species of the genus Pristiphora (Hymenoptera, Tenthredinidae) feeding on Norway spruce. Acta entomologica bohemoslovaca 73: 404-415.

- $\quad$ Benson RB (1935) The high mountain sawflies of Britain (Hymenoptera Symphyta). Transactions of the Royal Entomological Society of London 83 (1): 23-39. DOI: 10.1111/ j.1365-2311.1935.tb00412.x

- $\quad$ Benson RB (1943a) Some reputed British sawflies not found since Stephens's days (Hym., Symphyta). Entomologist's Monthly Magazine 79: 5-7.

- $\quad$ Benson RB (1943b) More about the sawflies of Hertfordshire and Buckinghamshire and the list of British species (Hym., Symphyta). Entomologist's Monthly Magazine 79: 7-12.

- Benson RB (1951) Hymenoptera, Symphyta. Handbooks for the Identification of British Insects. 6(2a). Royal Entomological Society, 49 pp.

- $\quad$ Benson RB (1952) Hymenoptera, Symphyta. Handbooks for the Identification of British Insects. 6(2b). Royal Entomological Society, 51-137 pp.

- Benson RB (1954) British sawfly galls of the genus Nematus [Pontania] on Salix. (Hymenoptera, Tenthredinidae). Journal of the Society for British Entomology 4: 206-211.

- $\quad$ Benson RB (1958) Hymenoptera, Symphyta. Handbooks for the Identification of British Insects. 6(2c). Royal Entomological Society, 139-258 pp.

- Benson RB (1968) Hymenoptera from Turkey, Symphyta. Bulletin of the British Museum (Natural History), Entomology 22: 111-207.

- Blank SM (1998) Die mittel- und nordeuropäischen Selandriinae (Hymenoptera: Tenthredinidae). In: Taeger A, Blank SM (Eds) Pflanzenwespen Deutschlands (Hymenoptera, Symphyta). Kommentierte Bestandsaufnahme. Goecke \& Evers, Keltern, 17 pp.

- $\quad$ Blank SM, Taeger A (1998) Comments on the taxonomy of Symphyta (Hymenoptera) (Preliminary studies for a catalogue of Symphyta, part 4). In: Taeger A, Blank SM (Eds) Pflanzenwespen Deutschlands (Hymenoptera, Symphyta). Kommentierte Bestandsaufnahme. Goecke \& Evers, Keltern, 141-174 pp.

- $\quad$ Blank SM, Groll EK, Liston AD, Prous M, Taeger A (2012) ECatSym - Electronic World Catalog of Symphyta (Insecta, Hymenoptera). 4.0 beta, data version 39. Digital Entomological Information, Müncheberg. Release date: 201212 18. URL: http:// sdei.senckenberg.de/ecatsym/ 
- Blank SM, Taeger A, Liston AD, Smith DR, Rasnitsyn AP, Shinohara A, Heidemaa M, Viitasaari M (2009) Studies toward a World Catalog of Symphyta (Hymenoptera). Zootaxa 2254: 1-96. URL: http://www.mapress.com/zootaxa/list/2009/zt02254.html

- Blank SM, Deters S, Drees M, Jänicke M, Jansen E, Kraus M, Liston AD, Ritzau C, Taeger A (2001) Symphyta. In: Dathe HH, Taeger A, Blank SM (Eds) Verzeichnis der Hautflügler Deutschlands (Entomofauna Germanica 4). Entomologische Nachrichten und Berichte. Beiheft 7. 19 pp.

- Bold TJ (1873) Capture in Northumberland of a Saw-fly new to Britain. Entomologist's Monthly Magazine 10: 69.

- Brischke CG (1884) Beobachtungen über die Arten der Blatt- und Holzwespen von C. G. A. Brischke, Hauptlehrer a. D. in Langfuhr und Dr. Gustav Zaddach, Professor in Königsberg, mitgetheilt von Brischke aus Zaddachs Manuskripten [Schluss, Nematus Teil 3]. Schriften der physikalisch-ökonomischen Gesellschaft zu Königsberg 24[1883]: 121-173.

- $\quad$ Broad GR (2014) Checklist of British and Irish Hymenoptera - Introduction. Biodiversity Data Journal 2: e1113. DOI: 10.3897/BDJ.2.e1113

- Cameron P (1882) A Monograph of the British Phytophagous Hymenoptera. (Tenthredo, Sirex and Cynips, Linné.). London, 1: 1-340; plates 1-21.

- Chevin H (1980) Notes sur les Hymenopteres Tenthredoides. Bulletin mensuel de la Société Linnéenne de Lyon 49: 453-456.

- $\quad$ Edmunds R, Hind S, Liston A, Palmer K (2007) Parna apicalis (Brischke, 1888) (Symphyta:Tenthredinidae), a leaf-mining sawfly new to Great Britain. Entomologist's Record and Journal of Variation 119: 223-226.

- Enslin E (1912) Die Tenthredinoidea Mitteleuropas. Deutsche Entomologische Zeitschrift Beiheft 1: 1-98. [In German].

- Enslin E (1913) Die Tenthredinoidea Mitteleuropas II. Deutsche Entomologische Zeitschrift Beiheft 2: 99-202. [In German].

- Enslin E (1914) Die Tenthredinoidea Mitteleuropas III. Deutsche Entomologische Zeitschrift Beiheft 3: 203-310. [In German].

- Enslin E (1915) Die Tenthredinoidea Mitteleuropas IV. Deutsche Entomologische Zeitschrift Beiheft 4: 311-412. [In German].

- Enslin E (1916) Die Tenthredinoidea Mitteleuropas V. Deutsche Entomologische Zeitschrift Beiheft 5: 413-538. [In German].

- Enslin E (1917) Die Tenthredinoidea Mitteleuropas VI. Deutsche Entomologische Zeitschrift Beiheft 6: 539-662. [In German].

- Enslin E (1918) Die Tenthredinoidea Mitteleuropas VII. (Schluß). Deutsche Entomologische Zeitschrift Beiheft 7: 663-790. [In German].

- Fabricius JC (1781) Species Insectorum exhibentes eorum differentias specificas, synonyma auctorum, loca natalia, metamorphosin adiectis observationibus, descriptionibus. 1. Ernest Bohnii, Hamburgi et Kilonii, I-VIII, 1-552 pp. URL: http:// www.biodiversitylibrary.org/item/44032\#page/7/mode/1up

- Fitton MG, Graham MWRdV, Bouček ZRJ, Fergusson NDM, Huddleston T, Quinlan J, Richards OW (1978) Kloet and Hincks. A check list of British insects. Part 4: Hymenoptera. Handbooks for the Identification of British Insects. 11(4). Royal Entomological Society, ix +159 pp. 
- $\quad$ Gibbs D (2006) Rose Bud Sawfly, Monardis plana (Klug) (Hymenoptera:

Tenthridinidae), new to Britain, discovered in South Wales. British Journal of Entomology and Natural History 19: 105-108.

- Goulet H (1986) The genera and species of the Nearctic Dolerini (Symphyta:

Tenthredinidae: Selandriinae): Classification and Phylogeny. Memoirs of the

Entomological Society of Canada 118 (135): 5-208. DOI: 10.4039/entm118135fv

- Grearson KJ (2006) A sawfly, Pristiphora leucopus (Hellén), (Hymenoptera:

Tenthredinidae) new to Britain. British Journal of Entomology and Natural History 19 (1): 1-5.

- Halstead AJ (2004) Berberis Sawfly, Arge berberidis Schrank (Hymenoptera: Argidae), a pest new to Britain. British Journal of Entomology and Natural History 17: 131-135.

- Haris A (2000) Study on the Palaearctic Dolerus Panzer, 1801 species (Hymenoptera: Tenthredinidae). Folia Entomologica Hungarica 61: 95-148.

- Haris A (2006) Study on the Palaearctic Pristiphora species (Hymenoptera: Tenthredinidae). Natura Somogyiensis 9: 201-277. URL: http://www.smmi.hu/termtud/ ns/ns9/Haris\%20NS9-2.pdf

- Heidemaa M (2004) Systematic studies on sawflies of the genera Dolerus, Empria and Caliroa (Hymenoptera: Tenthredinidae). Dissertationes Biologicae Universitatis Tartuensis 99: 1-162.

- Heidemaa M, Viitasaari M (1999) Taxonomy of Empria hungarica species-group (Hymenoptera, Tenthredinidae) in Northern Europe. Entomologica Fennica 10: 95-101.

- Heidemaa M, Nuorteva M, Hantula J, Saarma UM (2004) Dolerus asper Zaddach, 1859 and Dolerus brevicornis Zaddach, 1859 (Hymenoptera: Tenthredinidae), with notes on their phylogeny. European Journal of Entomology 101 (4): 637-650. DOI: $10.14411 /$ eje.2004.087

- ICZN (1999) International Code of Zoological Nomenclature. Fourth Edition. The International Trust for Zoological Nomenclature c/o The Natural History Museum, London, $306 \mathrm{pp}$.

- $\quad$ ICZN (2005) Opinion 2101 (Case 3243). Lyda latifrons Fallén, 1808 and L. gyllenhali Dahlbom, 1835 (currently Pamphilius latifrons and P. gyllenhali; Insecta, Hymenoptera): usage of the specific names conserved by designation of a neotype for Lyda latifrons. Bulletin of Zoological Nomenclature 62: 49-50. URL: http://biodiversitylibrary.org/ page/34357505

- $\quad$ Knight GT (2004) Kaliofenusa pusilla (Serville 1823) (Hymenoptera: Tenthredinidae). A species of leaf-mining sawfly new to Ireland. Bulletin of the Irish Biogeographical Society 28: 61-62.

- Knight GT (2006) First British records of the sawfly Emphytus laticinctus (Serville) (Hymenoptera: Tenthredinidae). British Journal of Entomology and Natural History 19: 193-198.

- Knight GT (2009) Empria basalis Lindqvist, 1968 - a new sawfly to Britain. Sawfly Study Group Newsletter 4: 15-16.

- $\quad$ Koch F (1988) Die Gattung Claremontia Rohwer (Hymenoptera, Tenthredinidae). Deutsche entomologische Zeitschrift 35: 311-330. DOI: 10.1002/mmnd.19880350421

- Konow FW (1899) Neuer Beitrag zur Synonymie der Chalastogastra. Entomologische Nachrichten (F. Karsch), Berlin 25 (6): 86-96. [In German]. URL: http:// www.biodiversitylibrary.org/ia/entomologischena251899berl\#page/116/mode/1up

- $\quad$ Konow FW (1905) Hymenoptera. Fam. Tenthredinidae. Genera Insectorum 29: 1-176. 
- Kopelke J (1991) Die Arten der viminalis-Gruppe, Gattung Pontania O. Costa 1859, Mittel- und Nordeuropas (Insecta: Hymenoptera: Tenthredinidae). Senckenbergiana biologica 71: 65-128.

- Kopelke J (1996) Die Euura atra- und amerinae-Gruppe in Nord- und Mitteleuropa (Insecta: Hymenoptera: Tenthredinidae: Nematinae). Senckenbergiana biologica 76: 93-113.

- Kopelke J (2000) Euura auritae sp. n. - ein neuer Gallenerzeuger der atra-Gruppe in Europa (Insecta, Hymenoptera, Tenthredinidae, Nematinae). Senckenbergiana biologica 80: 159-163.

- Kopelke J (2001) Die Artengruppen von Euura mucronata und E. laeta in Europa (Insecta, Hymenoptera, Tenthredinidae, Nematinae). Senckenbergiana biologica 81: 191-225.

- Kopelke J (2003) Gall-forming Nematinae, their willow hosts (Salix spec.) and biological strategies (Insecta, Hymenoptera, Symphyta, Tenthredinidae, Nematinae: Euura, Phyllocolpa, Pontania). Senckenbergiana biologica 82: 163-189.

- Kopelke J (2007a) The European species of the genus Phyllocolpa, part II: the leucapsis-group (Insecta, Hymenoptera, Tenthredinidae, Nematinae). Senckenbergiana biologica 87: 149-161.

- Kopelke J (2007b) The European species of the genus Phyllocolpa, part III: the species groups of crassispina, scotaspis, and piliserra (Insecta, Hymenoptera, Tenthredinidae, Nematinae). Senckenbergiana biologica 87: 163-183.

- Kopelke J (2007c) The European species of the genus Phyllocolpa, part I: the leucosticta-group (Insecta, Hymenoptera, Tenthredinidae, Nematinae).

Senckenbergiana biologica 87: 75-109.

- Lacourt J (1987) Note sur Pristiphora pallidiventris (Fallén) (= Pristiphora denudata Konow) avec description de deux nouvelles sous-espèces (Hymenoptera, Tenthredinidae). Nouvelle Revue d'Entomologie 4: 259-264.

- Lacourt J (1999) Répertoire des Tenthredinidae ouest-paléarctiques (Hymenoptera, Symphyta). Mémoires de la Société Entomologique de France 3: 1-432.

- Lacourt J (2000) Liste des espèces de la famille des Tenthredinidae décrites par J. G. Audinet-Serville, en Mai 1823 et par A. L. M. Le Peletier Comte de Saint-Fargeau, en Août 1823, avec désignation de lectotypes [Hymenoptera, Symphyta]. Revue francaise d'Entomologie (N. S.) 22: 77-108.

- Leach WE (1817) The Zoological Miscellany. Being Descriptions of New or Interesting Animals. 3. R. and A. Taylor, London, 1-151 pp. URL: http://biodiversitylibrary.org/ page/28686803\#page/7/mode/1up

- Leppänen S, Altenhofer E, Liston A, Nyman T (2012) Phylogenetics and evolution of host-plant use in leaf-mining sawflies (Hymenoptera: Tenthredinidae: Heterarthrinae). Molecular Phylogenetics and Evolution 64 (2): 331-341. DOI: 10.1016/ j.ympev.2012.04.005

- Lindqvist E (1955) Über einige Pristiphora-Arten aus Fennoskandien (Hym., Tenthredinidae). Notulae Entomologicae 35: 35-50.

- $\quad$ Lindqvist E (1962) Bemerkungen über paläarktische Blattwespen (Hym. Symph.). Notulae Entomologicae 42: 105-127.

- Lindqvist E (1972) Zur Nomenklatur und Taxonomie einiger Blattwespen (Hymenoptera, Symphyta). Notulae Entomologicae 52: 65-77. 
- $\quad$ Lindqvist E (1973) Taxonomische Bemerkungen über einige Blattwespen I (Hymenoptera, Symphyta). Notulae Entomologicae 53: 33-39.

- Liston AD (1981a) Pristiphora (Lygaeophora) lanifica (Zaddach, 1882) new to Britain (Hym., Tenthredinidae). Entomologist's Gazette 32: 181-184.

- $\quad$ Liston AD (1981b) Notes on little known British Pristiphora Lat. (Hym., Tenthredinidae) of the abietina-group. Entomologist's Monthly Magazine 117: 73-75.

- Liston AD (1982) Some sawflies from Whitlaw Moss Nature Reserve, Southern Scotland, with a species new to Britain (Hym.: Symphyta). Entomologist's Record and Journal of Variation 94: 175-179.

- $\quad$ Liston AD (1983a) Pristiphora karvoneni (Lindqvist) (Hym., Tenthredinidae) new to Britain. Entomologist's Monthly Magazine 119: 135-136.

- Liston AD (1983b) Distribution and Ecology of the Sawflies Macrophya alboannulata Costa and M. albicincta (Schrank) in the West Palaearctic. Faunistische Abhandlungen 10: 151-153.

- $\quad$ Liston AD (1985) On some sawflies (Hymenoptera, Symphyta) from Upper Deeside. Entomologist's Record and Journal of Variation 97: 94-95.

- Liston AD (1993) Caliroa tremulae Chevin in the British Isles and Central Europe (Hymenoptera: Tenthredinidae). Entomologist 112: 67-68.

- Liston AD (1994) Kaliofenusa carpinifoliae Liston (Hymenoptera: Tenthredinidae), a newly recognized leaf-miner on Field Elms in Britain. British Journal of Entomology and Natural History 7: 15-18.

- $\quad$ Liston AD (1995) Compendium of European Sawflies. List of species, modern nomenclature, distribution, foodplants, identification literature. Chalastos Forestry, Gottfrieding, 1-190 pp.

- Liston AD (1996) Compendium of European Sawflies. Supplement. Chalastos Press, Gottfrieding.

- Liston AD (2007) Revision of Stauronematus Benson, 1953 and additions to the sawfly fauna of Corsica and Sardinia. Beiträge zur Entomologie 57: 135-150.

- Liston AD, Blank SM (2006) New and little-known British Xyelidae and Tenthredinidae (Hymenoptera, Symphyta). Entomologist's Monthly Magazine 142: 219-227.

- $\quad$ Liston AD, O'Connor JP (2005) Records of Irish sawflies (Hymenoptera: Symphyta) including eight species new to Ireland. Irish Naturalist's Journal 28: 159-161.

- $\quad$ Liston AD, Prous M (2014) Sawfly taxa (Hymenoptera, Symphyta) described by Edward Newman and Charles Healy. ZooKeys 398: 83-98. DOI: 10.3897/zookeys.398.6595

- $\quad$ Liston AD, Taeger A, Blank SM (2006) Comments on European Sawflies (Hymenoptera: Symphyta). In: Blank SM, Schmidt S, Taeger A (Eds) Recent Sawfly Research: Synthesis and Prospects. Goecke \& Evers, Keltern.

- Liston AD, Knight GT, Heibo E, Bland KP, Barstad TE, Blank SM, Boevé J-, Fiedler K, Grearson KJ, Halstead A, Jacobs H-, Jansen E, Lønnve O, Prous M, Robinson J, Taeger A (2012) On Scottish sawflies, with results of the 14th International Sawfly Workshop, in the southern Highlands, 2010 (Hymenoptera, Symphyta). Beiträge zur Entomologie 62 (1): 1-68.

- $\quad$ Lorenz H, Kraus M (1957) Die Larvalsystematik der Blattwespen (Tenthredinoidea und Megalodontoidea). Abhandlungen zur Larvalsystematik der Insekten 1: 1-389.

- Malm T, Nyman T (2014) Phylogeny of the symphytan grade of Hymenoptera: new pieces into the old jigsaw(fly) puzzle. Cladistics online early: . 
- McLachlan R (1867) Additional notices respecting the maple-mining saw-fly (Phyllotoma aceris). Entomologist's Monthly Magazine 4: 123.

- Newman E (1838) Entomological notes. The Entomological Magazine 5[1837-1838] (2, 4, 5): 483-500.

- $\quad$ Nyman T (2002) The willow bud galler Euura mucronata Hartig (Hymenoptera: Tenthredinidae): one polyphage or many monophages? Heredity 88: 288-295. DOI: 10.1038/sj.hdy.6800042

- $\quad$ Nyman T, Zinovjev AG, Vikberg V, Farrel BD (2006) Molecular phylogeny of the sawfly subfamily Nematinae (Hymenoptera: Tenthredinidae). Systematic Entomology 31: 569-583. DOI: 10.1111/j.1365-3113.2006.00336.x

- O'Connor JP, Liston AD (1994) Pristiphora friesei (Konow) (Hymenoptera: Symphyta), a sawfly new to the British Isles. Entomologist's Gazette 45: 69-72.

- O'Connor JP, Liston AD, Speight MCD (1997) A Review of the Irish sawflies (Hymenoptera: Symphyta) including a checklist of species. Bulletin of the Irish Biogeographical Society 20: 2-99.

- Quinlan J (1978) Symphyta. In: Fitton MG, Graham MWRdV, Bouček ZRJ, Fergusson NDM, Huddleston T, Quinlan J, Richards OW (Eds) A check list of British insects. Part 4: Hymenoptera. Handbooks for the Identification of British Insects. 11(4). ix + 159 pp.

- Schiff NM, Goulet H, Smith DR, Boudreault C, Wilson AD, Scheffler BE (2012) Siricidae (Hymenoptera: Symphyta: Siricoidea) of the Western Hemisphere. Canadian Journal of Arthropod Identification 21: 1-305. DOI: 10.3752/cjai.2012.21

- $\quad$ Schmidt S (1997) Amauronematus Konow, 1890 - Ökologie und Taxonomie der nordeuropäischen Arten des fallax-Komplexes (Hymenoptera, Tenthredinidae). Beiträge zur Entomologie 47: 227-326.

- $\quad$ Shaw MR (2003) Adverse comment on the supposed British status of the web-spinning sawfly Cephalcia arvensis Panzer (Hymenoptera: Pamphiliidae). British Journal of Entomology and Natural History 16: 2.

- Shaw MR, Liston AD (1985) Xiphydria longicollis (Geoffroy) (Hymenoptera: Xiphydriidae) new to Britain. Entomologist's Gazette 36: 233-235.

- Sheppard DA (1990) A review of sawflies (Hymenoptera: Symphyta) from Jersey. Societe Jersiaise Annual Bulletin 25: 367-372.

- Shinohara A (1998) Pamphilius albopictus (Hymenoptera, Pamphiliidae) and its close relatives. Bulletin of the National Science Museum, Series A, Zoology 24 (4): 225-252. URL: http://ci.nii.ac.jp/naid/110004311889

- Shinohara A (2002) A spruce web-spinning sawfly, Cephalcia arvensis (Hymenoptera: Pamphiliidae) from Britain. British Journal of Entomology and Natural History 15: 134-135.

- Smith DR (1978) Suborder Symphyta. (Xyelidae, Pararchexyelidae, Parapamphiliidae, Xyelydidae, Karatavitidae, Gigasiricidae, Sepulcidae, Pseudosiricidae, Anaxyelidae, Siricidae, Xiphydriidae, Paroryssidae, Xyelotomidae, Blasticotomidae, Pergidae). Hymenopterorum Catalogus (nova editio) 14: 1-193.

- Stephens JF (1835) Illustrations of British Entomology; or, a Synopsis of Indigenous Insects: containing their generic and specific distinctions; with an account of their metamorphosis, times of appearance, localities, food, and economy, as far as practicable. Mandibulata. 7. Baldwin \& Cradock, London, 1-312, plates XXXV-XLVII pp. URL: http://www.biodiversitylibrary.org/item/97241\#page/5/mode/1up 
- Taeger A (1998) Bestimmungsschlüssel der Keulhornblattwespen Deutschlands (Hymenoptera: Cimbicidae). In: Taeger A, Blank SM (Eds) Pflanzenwespen Deutschlands (Hymenoptera, Symphyta). Kommentierte Bestandsaufnahme. Goecke \& Evers, Keltern, $12 \mathrm{pp}$.

- Taeger A (2002) The Megalodontesidae of Europe (Hymenoptera, Symphyta). In: Viitasaari M (Ed.) Sawflies (Hymenoptera, Symphyta) I. A review of the suborder, the Western Palaearctic taxa of Xyeloidea and Pamphilioidea. Tremex, Helsinki, 516 pp. [ISBN 952-5274-01-2].

- $\quad$ Taeger A, Blank SM (1996) Kommentare zur Taxonomie der Symphyta (Hymenoptera) (Vorarbeiten zu einem Katalog der Pflanzenwespen, Teil 1). Beiträge zur Entomologie 46: 251-275. [In German].

- $\quad$ Taeger A, Blank SM (1998) Beitrag zur Kenntnis einiger Nematinae (Hymenoptera: Tenthredinidae). In: Taeger A, Blank SM (Eds) Pflanzenwespen Deutschlands (Hymenoptera, Symphyta). Kommentierte Bestandsaufnahme. Goecke \& Evers, Keltern, $30 \mathrm{pp}$.

- $\quad$ Taeger A, Blank SM (2006) Bibliographic Notes. In: Blank SM, Schmidt S, Taeger A (Eds) Recent Sawfly Research: Synthesis and Prospects. Goecke \& Evers, Keltern, 319-330 pp.

- $\quad$ Taeger A, Blank SM, Liston AD (2010) World Catalog of Symphyta (Hymenoptera). Zootaxa 2580: 1-1064. URL: http://www.mapress.com/zootaxa/list/2010/2580.html

- Viitasaari M (1985) Taxonomic notes on the genus Trichiosoma Leach (Hymenoptera, Cimbicidae) I. Annales Entomologici Fennici 51: 25-26.

- Viitasaari M (1989) Taxonomic notes on the genus Trichiosoma Leach (Hymenoptera, Cimbicidae) II. Annales Entomologici Fennici 55: 111-129.

- Viitasaari M, Midtgaard F (1989) A contribution to the taxonomy of horntails with notes on the genus Sirex Linnaeus (Hymenoptera, Siricidae). Annales Entomologici Fennici 55: 103-110.

- Vikberg V (2003) Taxonomy of the species of Eupontania crassipes- and aquilonisgroups (Hymenoptera: Tenthredinidae: Nematinae). Entomologica Fennica 14: 129-172.

- Vikberg V (2004) Seasonal head dimorphism and taxonomy of some European species of Aprosthema (Hymenoptera: Symphyta: Argidae). Beiträge zur Entomologie 54: 107-125.

- Vikberg V (2006) Pristiphora Latreille, 1810 (Hymenoptera: Tenthredinidae: Nematinae) - two closely related species feeding on Lathyrus and Salix and notes on species feeding on leguminous plants. In: Blank SM, Schmidt S, Taeger A (Eds) Recent Sawfly Research: Synthesis and Prospects. Goecke \& Evers, Keltern, 23 pp.

- Vikberg V (2010a) European species of Tubpontania gen. nov., a new genus for species of the Pontania crassispina group (Hymenoptera: Tenthredinidae: Nematinae). Zootaxa 2620: 1-28.

- Vikberg V (2010b) On the taxonomy of Phyllocolpa scotaspis (Förster, 1854) and Phyllocolpa anglica (Cameron, 1877) and notes on the species groups of Phyllocolpa (Hymenoptera: Tenthredinidae: Nematinae). Sahlbergia 15: 3-13.

- Vikberg V, Liston AD (2009) Taxonomy and biology of European Heptamelini (Hymenoptera, Tenthredinidae, Selandriinae). Zootaxa 2112: 1-24.

- Vikberg V, Malinen P (2012) On the identity of Pontania dolichura (Thomson, 1871) and Pontania femoralis (Cameron, 1876) (Hymenoptera: Tenthredinidae: Nematinae).

Sahlbergia 18 (1): 25-39. 
- Vikberg V, Zinovjev A (2006) On the taxonomy and the host plants of North European species of Eupontania (Hymenoptera: Tenthredinidae: Nematinae). Beiträge zur Entomologie 56: 239-268.

- Zhelochovtsev AN (1988) Pereponchatokrylye. Shestaya chast. In: Medvedev GC (Ed.) Opredelitel' nasekomykh evropeyskoy chasti SSSR. 3 (6). Nauka, Leningrad, 3-237 pp. [In Russian]. 